\title{
Uniform Attractors for a Phase-field Model with Memory and Quadratic Nonlinearity
}

\section{Claudio Giorgi, Maurizio Grasselli E. VitToRino PATA}

\begin{abstract}
A phase-field system with memory which describes the evolution of both the temperature variation $\vartheta$ and the phase variable $\chi$ is considered. This thermodynamically consistent model is based on a linear heat conduction law of Coleman-Gurtin type. Moreover, the internal energy linearly depends both on the present value of $\vartheta$ and on its past history, while the dependence on $\chi$ is represented through a function with quadratic nonlinearity. A Cauchy-Neumann initial and boundary value problem associated with the evolution system is then formulated in a history space setting. This problem is shown to generate a non-autonomous dynamical system which possesses a uniform attractor. In the autonomous case, the attractor has finite Hausdorff and fractal dimensions whenever the internal energy linearly depends on $\chi$.
\end{abstract}

\section{INTRODUCTION}

In this paper we investigate the well-posedness and the long time behavior of a phase-field model with thermal memory based on a heat conduction law of Coleman-Gurtin type [CG]. In a previous paper [GGP], the authors considered a similar problem, after constructing the model equations on the basis of the hereditary heat conduction theory of Gurtin and Pipkin [GuP]. The approach we adopt here mainly differs from [GGP] for the heat flux vector depends on the present value of the temperature gradient, besides its past history. As a consequence, in the present framework a Fourier heat conduction law is recovered when all memory terms are neglected. 
Let $\Omega \subset \mathbb{R}^{3}$ be a fixed bounded domain occupied by a rigid, isotropic, and homogeneous heat conductor, undergoing some temperature-dependent phase transition. Along the lines of [GGP] (cf., in particular, Appendix) we consider only small variations of the absolute temperature and its gradient, and we suppose that at each point $x \in \Omega$ the state of the material is described by the triplet $\left(\vartheta, \vartheta^{t}, \chi\right)$. Here $\vartheta(x, t)$ is the temperature variation field from the reference value $\theta_{c}$ (at which transition occurs), namely (cf. Appendix in [GGP])

$$
\vartheta=\frac{\theta-\theta_{c}}{\theta_{c}}
$$

where $\theta$ is the absolute temperature. Moreover, $\vartheta^{t}(x, s)=\vartheta(x, t-s), s \geq 0$, is the past history of $\vartheta$ up to time $t$, and $\chi(x, t)$ is the phase variable, describing the kinetics of the solid-liquid transition.

The evolution of the temperature-dependent phase change phenomenon is governed by the energy balance equation

$$
\partial_{t} e+\operatorname{div} \mathbf{q}=f
$$

where $e$ is the internal energy, $\mathbf{q}$ is heat flux vector, and $f$ is the external heat source, along with some phase-field relationship. We will adopt a phase-field equation of the Caginalp type (see [Cag])

$$
m \partial_{t} \chi-m_{0} \Delta \chi+m_{1} \chi^{3}=\gamma(\chi)+\lambda^{\prime}(\chi) \vartheta
$$

where $m, m_{0}$, and $m_{1}$ are positive constants, while $\lambda$ and $\gamma$ are smooth functions.

Equation (1.2) can be obtained following two different, but thermodynamically consistent strategies. If we adopt the Penrose-Fife point of view [PF], then (1.2) represents a sort of constitutive equation for the internal variable $\chi$, which generalizes the Ginzburg-Landau equation to dynamical temperature-dependent phenomena (see also [BS]). As shown in the Appendix of [GGP], this approach is unaffected by the introduction of the past history of $\vartheta$ into the state variables. Thus, using the Clausius-Duhem inequality, and linearizing in $\vartheta$, a thermodynamically consistent system containing equation (1.2) is obtained.

Alternatively, according to Gurtin-Fried theory [GF], the phase-field equation (1.2) can be deduced, by linearization in $\vartheta$, from a balance equation involving new constitutive quantities, called accretive sources and fluxes, which take into account microscopic actions due to phase changes.

Since compatibility of (1.2) with hereditary constitutive equations is still an open question, we adopt here the former approach. Thus, taking into consideration a linearized version of the Coleman-Gurtin theory [CG] and paralleling the procedure outlined in [GGP], we assume that $e$ and $\mathbf{q}$ are described by the 
following constitutive equations

$$
\begin{aligned}
& e(x, t)=e_{c}+c_{v} \theta_{c} \vartheta(x, t)+\int_{0}^{\infty} a(\sigma) \vartheta(x, t-\sigma) d \sigma+\theta_{c} \lambda(\chi(x, t)) \\
& \mathbf{q}(x, t)=-k_{I} \nabla \vartheta(x, t)-\int_{0}^{\infty} k(\sigma) \nabla \vartheta(x, t-\sigma) d \sigma
\end{aligned}
$$

for $(x, t) \in \Omega \times \mathbb{R}$, where $e_{c}>0, c_{v}>0$, and $k_{I}>0$ are the internal energy at equilibrium, the specific heat, and the instantaneous heat conductivity, respectively. Moreover, we require that the memory kernels $k$ and $a$ are smooth enough and summable on $(0,+\infty), k$ and $a^{\prime}$ vanish at infinity, and $a(0)>0$.

In virtue of (1.3), the energy balance (1.1) reads

$$
\begin{gathered}
c_{v} \theta_{c} \partial_{t} \vartheta+a(0) \vartheta+\int_{0}^{\infty} a^{\prime}(\sigma) \vartheta(t-\sigma) d \sigma+\theta_{c} \lambda^{\prime}(\chi) \partial_{t} \chi \\
-k_{I} \Delta \vartheta-\int_{0}^{\infty} k(\sigma) \Delta \vartheta(t-\sigma) d \sigma=f .
\end{gathered}
$$

Concerning initial conditions, we have to specify initial values at a given time $\tau \in \mathbb{R}$ for all the state variables. Thus, due to the presence of memory dependent terms into the constitutive equations, besides the values of $\vartheta$ and $\chi$ at $\tau$, the whole past history of $\vartheta$ up to $\tau$ must be given, namely

$$
\begin{aligned}
\vartheta(\tau) & =\vartheta_{0} & & \text { in } \Omega \\
\chi(\tau) & =\chi_{0} & & \text { in } \Omega \\
\vartheta(\tau-s) & =\vartheta_{0}(s) & & \text { in } \Omega, \text { for all } s>0
\end{aligned}
$$

where $\vartheta_{0}(s)$ is the initial past history of $\vartheta$.

Quite natural boundary conditions for the phase-field system (1.2), (1.4) are given assuming that the fluxes of both heat and phase-field gradients vanish across the boundary. Namely, for $\chi$ we have

$$
\partial_{\mathbf{n}} \chi=0 \quad \text { on } \partial \Omega \times(\tau,+\infty)
$$

$\partial_{\mathbf{n}}$ being the usual outward normal derivative, while the adiabatic boundary condition $\left.\mathbf{q} \cdot \mathbf{n}\right|_{\partial \Omega}=0$ is expressed by the integral equation

$$
k_{I} \partial_{\mathbf{n}} \vartheta(t)+\int_{0}^{\infty} k(\sigma) \partial_{\mathbf{n}} \vartheta(t-\sigma) d \sigma=0 \quad \text { on } \partial \Omega \times(\tau,+\infty) .
$$


In order to reformulate the resulting initial and boundary value problem in a history space setting, we follow [GGP] (see also [Daf]) and we introduce the additional variable $\eta^{t}$, which is defined by

$$
\eta^{t}(x, s)=\int_{0}^{s} \vartheta^{t}(x, \tau) d \tau=\int_{t-s}^{t} \vartheta(x, \tau) d \tau \quad s>0 .
$$

This variable $\eta$ is easily seen to satisfy the equation

$$
\partial_{t} \eta^{t}(s)+\partial_{s} \eta^{t}(s)=\vartheta(t) \quad \text { in } \Omega,(t, s) \in(\tau,+\infty) \times(0,+\infty)
$$

along with the initial condition

$$
\eta^{\tau}=\eta_{0} \quad \text { in } \Omega \times(\tau,+\infty), \quad \text { where } \eta_{0}(s)=\int_{0}^{s} \vartheta_{0}(y) d y
$$

is the initial summed past history of $\vartheta$.

According to the assumed asymptotic behavior of kernels $k$ and $a^{\prime}$, we observe that formal integration by parts yields

$$
\int_{0}^{\infty} k(\sigma) \nabla \vartheta(t-\sigma) d \sigma=-\int_{0}^{\infty} k^{\prime}(\sigma) \nabla \eta^{t}(\sigma) d \sigma \quad \text { in } \Omega, t>\tau
$$

and

$$
\int_{0}^{\infty} a^{\prime}(\sigma) \vartheta(t-\sigma) d \sigma=-\int_{0}^{\infty} a^{\prime \prime}(\sigma) \eta^{t}(\sigma) d \sigma \quad \text { in } \Omega, t>\tau .
$$

Thus, setting

$$
\mu(s)=-k^{\prime}(s) \text { and } \nu(s)=-a^{\prime \prime}(s)
$$

for any $s>0$, and taking for simplicity all the (positive) constants equal to 1 , the above choice of variables leads to the following initial and boundary value problem.

Problem P. Find $(\vartheta, \chi, \eta)$ solution to the system

$\partial_{t}(\vartheta(t)+\lambda(\chi(t)))-\Delta \vartheta(t)+\vartheta(t)+\int_{0}^{\infty} \nu(\sigma) \eta^{t}(\sigma) d \sigma-\int_{0}^{\infty} \mu(\sigma) \Delta \eta^{t}(\sigma) d \sigma=f(t)$

$\partial_{t} \chi(t)-\Delta \chi(t)+\chi^{3}(t)=\gamma(\chi(t))+\lambda^{\prime}(\chi(t)) \vartheta(t)$

$\partial_{t} \eta^{t}(s)+\partial_{s} \eta^{t}(s)=\vartheta(t)$ 
in $\Omega$, for any $t>\tau$ and any $s>0$, which satisfies the initial and boundary conditions

$$
\begin{aligned}
\partial_{\mathbf{n}} \vartheta+\int_{0}^{\infty} \mu(\sigma) \partial_{\mathbf{n}} \eta^{t}(\sigma) d \sigma & =0 & & \text { on } \partial \Omega \times(\tau,+\infty) \\
\partial_{\mathbf{n}} \chi & =0 & & \text { on } \partial \Omega \times(\tau,+\infty) \\
\vartheta(\tau) & =\vartheta_{0} & & \text { in } \Omega \\
\chi(\tau) & =\chi_{0} & & \text { in } \Omega \\
\eta^{\tau} & =\eta_{0} & & \text { in } \Omega \times(0,+\infty) .
\end{aligned}
$$

Problems like $\mathbf{P}$ when $\lambda$ is a quadratic nonlinearity, which are also useful to describe ferromagnetic transformations (see, e.g., [HHM]), have been studied firstly in [BL1, BL2], where existence and uniqueness results have been proved via energy methods. In this framework, it is also worth quoting some papers which are devoted to the case $k_{I}=0$, assuming both $\gamma$ and $\lambda$ linear. Using a semigroup approach, existence, uniqueness, and longtime results have been proved firstly in $[\mathrm{AB}]$. Quite general well-posedness results have then been obtained in [CGG1, CGG2, CGG3] (cf. also [CGG4]) again via energy methods. Recently, in [CL1, CL2] a thorough investigation along the lines of [AB], for a more general model, has been carried out. In particular, existence and uniqueness when $\lambda$ is a quadratic nonlinearity as well as a detailed characterization of the $\omega$-limit set have been shown. Regarding the longtime behavior and existence of a maximal attractor for other phase transition models without memory effects, the reader is referred, e.g., to [Ken1, Ken2, KNZ, Lau, SZ] and references therein.

In all the mentioned papers about phase-field models with memory, the (summed) past history of $\vartheta$ (and, possibly, of $\chi$ ) is simply incorporated in the source term $f$ and, sometimes, in the boundary data. As a consequence, the Lyapunov stability analysis (i.e., with respect to perturbations of the initial data) does not involve the initial (summed) past history, which must be kept fixed (a different stability analysis, called structural stability, is required if we want perturb the past history of $\vartheta$, and then $f$ ). This approach seems not appropriate to study the longtime behavior of solutions from a more general point of view, namely, the stability of sets of trajectories under perturbation of all initial data. Instead, a formulation in the history space setting, which regards $\eta$ as a variable of the evolution phenomenon, has been proved to be effective in analyzing such issue (see [GGP, GMP2, PZ]). In particular, in [GGP] we have considered a problem similar to $\mathbf{P}$, but based on a heat conduction law with null istantaneous heat conductivity, i.e., $k_{I}=0$. This implies that the equation for $\vartheta$ does not contain the regularizing term $-\Delta \vartheta$ (cf. (1.4)). Working in the history space formulation, we have been able to prove, besides some well-posedness results, 
the existence of uniform absorbing sets. Here, taking advantage of the presence of $-\Delta \vartheta$, we can say more about the asymptotic behavior of the solutions to $\mathbf{P}$. Indeed, the main purpose of this work is to prove that problem $\mathbf{P}$ defines a strongly continuous process which possesses a uniform attractor.

The plan of the paper follows. In Section 2, we introduce some preliminary tools, while in Section 3 we formulate $\mathbf{P}$ in a proper functional setting, and we state well-posedness results, which are then proved in Section 4 and Section 5 . These results allow to express the solution to $\mathbf{P}$ in terms of a strongly continuous process of continuous operators. Section 6 is devoted to show the existence of a uniform absorbing set, as the time-dependent functional symbol $f$ is allowed to vary in a suitable Banach space. In Section 7, we present the main result, namely the existence of a uniform attractor for the process. In addition, when $\lambda$ is linear, we show in Section 8 that the attractor has finite fractal dimension.

Finally, it is worth noting that some thermal dissipation is supported by the hereditary term into the constitutive equation (1.3) for the internal energy. However, unlike the crucial role played by this term (and especially by $a(0)$ ) in the main result of [GGP] when $k_{I}=0$, here it merely compensates the absence of heat outflow due to the adiabatic (Neumann) boundary condition. As a consequence, all the results of the following sections remain still valid, with minor modifications, if we neglect either or both memory contributions into (1.4), provided that the Dirichlet boundary condition for the temperature is assumed rather than the Neumann one, so to exploit Poincaré inequality. From a physical point of view, this is quite natural, since such a boundary condition can act as a thermal pump in a dissipative way. Indeed, if the temperature inside grows beyond the value at the boundary, then the external environment removes heat from the boundary surface in order to keep fixed its temperature value.

It is interesting to observe that when all memory terms are neglected in the model equations and Dirichlet boundary condition for the temperature is considered, then a Fourier heat conduction law is recovered, and the state variables reduce to $\vartheta$ and $\chi$. Therefore $\mathbf{P}$ degenerates into the following initial and Dirichlet-Neumann boundary value problem.

Problem $\boldsymbol{P}^{\prime}$. Find $(\vartheta, \chi)$ solution to the system

$$
\begin{aligned}
\partial_{t}(\vartheta(t)+\lambda(\chi(t)))-\Delta \vartheta(t) & =f(t) \\
\partial_{t} \chi(t)-\Delta \chi(t)+\chi^{3}(t) & =\gamma(\chi(t))+\lambda^{\prime}(\chi(t)) \vartheta(t)
\end{aligned}
$$




$$
\begin{array}{rlrl}
\vartheta=0 & & \text { on } \partial \Omega \times(\tau,+\infty) \\
\partial_{\mathbf{n}} \chi=0 & & \text { on } \partial \Omega \times(\tau,+\infty) \\
\vartheta(\tau)=\vartheta_{0} & \text { in } \Omega \\
\chi(\tau)=\chi_{0} & \text { in } \Omega .
\end{array}
$$

Hence, we are dealing with a thermally induced phase-field model of the Caginalp type for which it is possible to prove well posedness as well as longtime behavior results analogous to those obtained for $\mathbf{P}$, included the existence of a uniform attractor.

\section{Terminology and Basic Tools}

Let $\Omega \subset \mathbb{R}^{3}$ be a bounded domain with smooth boundary $\partial \Omega$ (e.g., of class $\left.C^{2,1}\right)$. We set

$$
H=L^{2}(\Omega), \quad V=H^{1}(\Omega), \quad W=H^{2}(\Omega)
$$

and denote by $V^{*}$ the dual spaces of $V$. As usual, we identify $H$ with its dual space $H^{*}$ and we recall the compact and dense embeddings

$$
W \hookrightarrow V \hookrightarrow H \equiv H^{*} \hookrightarrow V^{*}
$$

We will always denote the norm and the inner product on a Hilbert space $\mathcal{X}$ by $\langle\cdot, \cdot\rangle_{\mathcal{X}}$ and $\|\cdot\|_{\mathcal{X}}$, respectively. Moreover, $\langle\cdot, \cdot\rangle_{\mathcal{X}}{ }_{\mathcal{X}}$ will indicate the duality pairing between $\mathcal{X}^{*}$ and $\mathcal{X}$.

Given a positive summable function $\alpha$ defined on $\mathbb{R}^{+}=(0,+\infty)$ and a real Hilbert space $\mathcal{X}$, let $L_{\alpha}^{2}\left(\mathbb{R}^{+}, \mathcal{X}\right)$ be the Hilbert space of $\mathcal{X}$-valued functions on $\mathbb{R}^{+}$, endowed with the inner product

$$
\left\langle\psi_{1}, \psi_{2}\right\rangle_{L_{\alpha}^{2}\left(\mathbb{R}^{+}, \mathcal{X}\right)}=\int_{0}^{\infty} \alpha(\sigma)\left\langle\psi_{1}(\sigma), \psi_{2}(\sigma)\right\rangle_{\mathcal{X}} d \sigma
$$

It is worth recalling that, given two Hilbert spaces $\mathcal{X}$ and $\mathcal{Y}$, the space $\mathcal{X} \cap \mathcal{Y}$ turns out to be a Hilbert space endowed with the inner product

$$
\langle\cdot, \cdot\rangle_{\mathcal{X} \cap \mathcal{Y}}=\langle\cdot, \cdot\rangle_{\mathcal{X}}+\langle\cdot, \cdot\rangle_{\mathcal{Y}}
$$

In order to describe the asymptotic behavior of the solutions to $\mathbf{P}$, we also need to introduce the Banach space $\mathcal{T}_{1}$ of $L_{\mathrm{loc}}^{1}$-translation bounded functions with 
values in $H$, namely,

$$
\mathcal{T}_{1}=\left\{f \in L_{\mathrm{loc}}^{1}(\mathbb{R}, H):\|f\|_{\mathcal{T}_{1}}=\sup _{r \in \mathbb{R}} \int_{r}^{r+1}\|f(y)\|_{H} d y<\infty\right\} .
$$

Similarly, we define the Banach space $\mathcal{T}_{2}$ of $L_{\text {loc }}^{2}$-translation bounded functions with values in $V^{*}$, that is,

$$
\mathcal{T}_{2}=\left\{f \in L_{\mathrm{loc}}^{2}\left(\mathbb{R}, V^{*}\right):\|f\|_{\mathcal{T}_{2}}^{2}=\sup _{r \in \mathbb{R}} \int_{r}^{r+1}\|f(y)\|_{V^{*}}^{2} d y<\infty\right\} .
$$

Definition 2.1. A function $g \in L_{\mathrm{loc}}^{1}(\mathbb{R}, H)$ is said to be translation compact in $L_{\mathrm{loc}}^{1}(\mathbb{R}, H)$ if the hull of $g$, defined as

$$
\mathrm{H}(g)=\overline{\left\{g^{r}\right\}_{r \in \mathbb{R}}} L_{\mathrm{loc}}^{1}(\mathbb{R}, H)
$$

is compact in $L_{\mathrm{loc}}^{1}(\mathbb{R}, H)$, where $g^{r}(\cdot)=g(\cdot+r)$ is the translate of $g$ by $r$.

The reader is referred to [CV3] and references therein for a more detailed presentation of the subject. Here we just recall that if $g$ is translation compact in $L_{\text {loc }}^{1}(\mathbb{R}, H)$, then $g \in \mathcal{T}_{1}$ and

$$
\|f\|_{\mathcal{T}_{1}} \leq\|g\|_{\mathcal{T}_{1}} \quad \text { for all } f \in \mathrm{H}(g) .
$$

We also remark that the class of translation compact functions in $L_{\text {loc }}^{1}(\mathbb{R}, H)$ is quite general. For example, it contains $L^{p}(\mathbb{R}, H)$ for all $p \geq 1$, the constant $H$-valued functions, and the class of almost periodic functions (see [AP]).

Throughout the paper, the symbol $c$ will stand for a generic positive constant, which may vary even in the same line within a proof. If not otherwise specified, $c$ will depend only on $\Omega, \nu, \mu, \gamma$, and $\lambda$.

Finally, for the reader's convenience, we report here below some technical results which will be useful in the course of the investigation, namely, a compactness lemma (see Lemma 5.5 in [PZ]), the uniform Gronwall lemma (see Lemma III.1.1 in [Tem]), and two Gronwall-type lemmas, which subsume Lemma A.5 in [Bre] and some results in [PPV].

Lemma 2.2. Let $\alpha \in C^{0}\left(\mathbb{R}^{+}\right) \cap L^{1}\left(\mathbb{R}^{+}\right)$be a non-negative non-increasing function, and let $\mathcal{X}_{0}, \mathcal{X}, \mathcal{X}_{1}$ be three Hilbert spaces such that

$$
\mathcal{X}_{0} \hookrightarrow \mathcal{X} \hookrightarrow \mathcal{X}_{1}
$$


the first injection being compact. Let $\mathcal{E} \subset L_{\alpha}^{2}\left(\mathbb{R}^{+}, \mathcal{X}\right)$ satisfy the following hypotheses:

(i) $\mathcal{E}$ is bounded in $L_{\alpha}^{2}\left(\mathbb{R}^{+}, \mathcal{X}_{0}\right) \cap H_{\alpha}^{1}\left(\mathbb{R}^{+}, \mathcal{X}_{1}\right)$

(ii) $\sup _{\eta \in \mathcal{E}}\|\eta(s)\|_{\mathcal{X}}^{2} \leq h(s) \quad$ a.e. for some $h \in L_{\alpha}^{1}\left(\mathbb{R}^{+}\right)$.

Then $\mathcal{E}$ is relatively compact in $L_{\alpha}^{2}\left(\mathbb{R}^{+}, \mathcal{X}\right)$.

Lemma 2.3. Let $\varphi, m_{1}$, and $m_{2}$ be three non-negative locally summable functions on $[\tau,+\infty)$ satisfying

$$
\frac{d}{d t} \varphi(t) \leq m_{1}(t) \varphi(t)+m_{2}(t) \text { for a.e. } t \in[\tau,+\infty)
$$

and such that

$$
\int_{t}^{t+1} m_{j}(s) d s \leq a_{j} \quad \text { and } \quad \int_{t}^{t+1} \varphi(s) d s \leq a_{3}
$$

$(j=1,2)$ for some positive constants $a_{1}, a_{2}, a_{3}$. Then

$$
\varphi(t+1) \leq\left(a_{2}+a_{3}\right) e^{a_{1}}
$$

for any $t \in[\tau,+\infty)$.

Lemma 2.4. Let $\varphi, m_{1}, m_{2}$, and $m_{3}$ be three non-negative summable functions on the interval $[\tau, T] \subset \mathbb{R}$. Then the differential inequality

$$
\frac{d}{d t} \varphi^{2}(t) \leq m_{1}(t) \varphi^{2}(t)+m_{2}(t) \varphi(t)+m_{3}(t) \text { for a.e. } t \in[\tau, T]
$$

implies

$$
\varphi^{2}(t) \leq 2 e^{\beta} \varphi^{2}(\tau)+e^{2 \beta}\left(\int_{\tau}^{t} m_{2}(y) d y\right)^{2}+2 e^{\beta} \int_{\tau}^{t} m_{3}(y) d y
$$

for any $t \in[\tau, T]$, where $\beta=\int_{\tau}^{T} m_{1}(y) d y$. 
Lemma 2.5. Let $\varphi, m_{1}$, and $m_{2}$ be three non-negative locally summable functions on $[\tau,+\infty)$ which satisfy, for some $\varepsilon>0$, the differential inequality

$$
\frac{d}{d t} \varphi^{2}(t)+\varepsilon \varphi^{2}(t) \leq m_{1}(t) \varphi(t)+m_{2}(t) \text { for a.e. } t \in[\tau,+\infty)
$$

Then

$$
\varphi^{2}(t) \leq 2 \varphi^{2}(\tau) e^{-\varepsilon(t-\tau)}+\left(\int_{\tau}^{t} m_{1}(y) e^{-\varepsilon / 2(t-y)} d y\right)^{2}+2 \int_{\tau}^{t} m_{2}(y) e^{-\varepsilon(t-y)} d y
$$

for any $t \in[\tau,+\infty)$. Moreover, the inequality

$$
\int_{\tau}^{t} m(y) e^{-\varepsilon(t-y)} d y \leq \frac{e^{\varepsilon}}{1-e^{-\varepsilon}} \sup _{r \geq \tau} \int_{r}^{r+1} m(y) d y
$$

holds for every non-negative locally summable function $m$ on $[\tau,+\infty)$ and every $\varepsilon>0$.

\section{WeLl-Posedness}

Before stating the main results, we must introduce a rigorous formulation of problem P. First of all, some assumptions are in order. As far as the kernels $\nu$ and $\mu$ are concerned, we suppose

(K1) $\nu, \mu \in C^{1}\left(\mathbb{R}^{+}\right) \cap L^{1}\left(\mathbb{R}^{+}\right)$

(K2) $\nu(s) \geq 0, \mu(s) \geq 0$ for all $s \in \mathbb{R}^{+}$

(K3) $\nu^{\prime}(s) \leq 0, \mu^{\prime}(s) \leq 0$ for all $s \in \mathbb{R}^{+}$

(K4) $\nu^{\prime}(s)+\delta \nu(s) \leq 0, \mu^{\prime}(s)+\delta \mu(s) \leq 0 \quad$ for some $\delta>0$, for all $s \in \mathbb{R}^{+}$.

Assumption (K4), which basically implies the exponential decay of the memory kernel (see, e.g., [GMP1]), is used only to prove the results related to longterm behavior (see Section 6 and Section 7 below). Then, we set

$$
a_{0}=\int_{0}^{\infty} \nu(\sigma) d \sigma \geq 0 \quad \text { and } \quad k_{0}=\int_{0}^{\infty} \mu(\sigma) d \sigma \geq 0
$$


and, in view of (K1)-(K2), we introduce the Hilbert spaces

$$
\begin{gathered}
\mathcal{N}=L_{\nu}^{2}\left(\mathbb{R}^{+}, V^{*}\right) \cap L_{\mu}^{2}\left(\mathbb{R}^{+}, H\right) \\
\mathcal{M}=L_{\nu}^{2}\left(\mathbb{R}^{+}, H\right) \cap L_{\mu}^{2}\left(\mathbb{R}^{+}, V\right) \\
\mathcal{L}=L_{\nu}^{2}\left(\mathbb{R}^{+}, V\right) \cap L_{\mu}^{2}\left(\mathbb{R}^{+}, W\right)
\end{gathered}
$$

and we denote

$$
\mathcal{H}=H \times V \times \mathcal{M} \quad \text { and } \quad \mathcal{V}=V \times W \times \mathcal{L} .
$$

Furthermore, we assume

$$
\begin{aligned}
\gamma & \in C^{1}(\mathbb{R}) \quad \text { and } \quad \gamma^{\prime} \in L^{\infty}(\mathbb{R}) \\
\lambda & \in C^{2}(\mathbb{R}) \quad \text { and } \quad \lambda^{\prime \prime} \in L^{\infty}(\mathbb{R}) \\
f & \in L_{\mathrm{loc}}^{1}(\mathbb{R}, H)+L_{\mathrm{loc}}^{2}\left(\mathbb{R}, V^{*}\right) \\
\vartheta_{0} & \in H \\
\chi_{0} & \in V \\
\eta_{0} & \in \mathcal{M} .
\end{aligned}
$$

Definition 3.1. Let (K1) and (K2) hold. Pick $\tau, T \in \mathbb{R}$ such that $T>\tau$ and set $I=[\tau, T]$. A triplet $(\vartheta, \chi, \eta)$ which fulfills

$$
\begin{aligned}
\vartheta & \in C^{0}(I, H) \cap L^{2}(I, V) \\
\vartheta_{t} & \in L^{2}\left(I, V^{*}\right)+L^{1}(I, H) \\
\chi & \in H^{1}(I, H) \cap C^{0}(I, V) \cap L^{2}(I, W) \\
\eta & \in C^{0}(I, \mathcal{M}) \\
\eta_{t}+\eta_{s} & \in L^{2}(I, \mathcal{M}) \cap C^{0}(I, \mathcal{N})
\end{aligned}
$$




$$
\begin{aligned}
\left\langle\vartheta_{t}, v\right\rangle_{V^{*} V}+\left\langle\lambda^{\prime}(\chi) \chi_{t}, v\right\rangle_{H} & +\langle\vartheta, v\rangle_{V}+\int_{0}^{\infty} \nu(\sigma)\langle\eta(\sigma), v\rangle_{H} d \sigma \\
+\int_{0}^{\infty} \mu(\sigma)\langle\nabla \eta(\sigma), \nabla v\rangle_{H^{3}} d \sigma & =\langle f, v\rangle_{V^{*} V} \quad \text { for all } v \in V, \text { a.e. in } I \\
\chi_{t}-\Delta \chi+\chi^{3} & =\gamma(\chi)+\lambda^{\prime}(\chi) \vartheta \quad \text { a.e. in } \Omega \times I \\
\left\langle\eta_{t}+\eta_{s}, \psi\right\rangle_{\mathcal{M}} & =\langle\vartheta, \psi\rangle_{\mathcal{M}} \quad \text { for all } \psi \in \mathcal{M}, \text { a.e. in } I \\
\partial_{\mathbf{n}} \chi & =0 \quad \text { a.e. on } \partial \Omega \times I \\
\vartheta(\tau) & =\vartheta_{0} \quad \text { a.e. in } \Omega \\
\chi(\tau) & =\chi_{0} \quad \text { a.e. in } \Omega \\
\eta^{\tau} & =\eta_{0} \quad \text { a.e. in } \Omega \times \mathbb{R}^{+} .
\end{aligned}
$$

The well-posedness of $\mathbf{P}$ is ensured by the following:

Theorem 3.2. Let (K1)-(K3) and (H1)-(H6) hold. Then, given any initial time $\tau \in \mathbb{R}$ and any $T>\tau$, problem $\mathbf{P}$ has a unique solution $(\vartheta, \chi, \eta)$ in the interval $I=[\tau, T]$. Moreover, let $\left\{f_{i}, \vartheta_{0 i}, \chi_{0 i}, \eta_{0 i}\right\}, i=1,2$, be two sets of data satisfying (H3)-(H6), indicate by $\left\{\vartheta_{i}, \chi_{i}, \eta_{i}\right\}$ the corresponding solutions to problem $\mathbf{P}$, and set

$$
\begin{array}{cl}
\omega_{i}=\vartheta_{i}+\lambda\left(\chi_{i}\right) & \text { a.e. in } \Omega \times I \\
\omega_{0 i}=\vartheta_{0 i}+\lambda\left(\chi_{0 i}\right) & \text { a.e. in } \Omega .
\end{array}
$$

Then there exists a positive constant $\Lambda_{1}$ such that, for any $t \in I$,

$$
\begin{gathered}
\left\|\omega_{1}(t)-\omega_{2}(t)\right\|_{V^{*}}^{2}+\int_{\tau}^{t}\left\|\omega_{1}(y)-\omega_{2}(y)\right\|_{H}^{2} d y+\left\|\chi_{1}(t)-\chi_{2}(t)\right\|_{H}^{2} \\
+\int_{\tau}^{t}\left\|\nabla \chi_{1}(y)-\nabla \chi_{2}(y)\right\|_{H^{3}}^{2} d y+\left\|\eta_{1}^{t}-\eta_{2}^{t}\right\|_{\mathcal{N}}^{2} \\
\leq \Lambda_{1}\left(\left\|\omega_{01}-\omega_{02}\right\|_{V^{*}}^{2}+\left\|\chi_{01}-\chi_{02}\right\|_{H}^{2}+\left\|\eta_{01}-\eta_{02}\right\|_{\mathcal{N}}^{2}+\left\|f_{1}-f_{2}\right\|_{L^{1}\left(I, V^{*}\right)}^{2}\right) .
\end{gathered}
$$

Remark 3.3. As we shall see in Sec. 3, existence and uniqueness can still be proved even if the term $\chi^{3}$ in equation (3.7) is replaced by a maximal monotone graph $\beta$ in $\mathbb{R}^{2}$, provided that $\varphi\left(\chi_{0}\right) \in L^{1}(\Omega)$, where $\varphi: \mathbb{R} \rightarrow[0,+\infty]$ is the 
proper, convex, and lower-semicontinuous function whose subdifferential coincides with $\beta$ (see [BL1] and [GGP]). Also, it is worth noting that the existence proof can be extended to functions $\lambda$ with cubic growth (see Section 4 below and Remark 4.1 in [BL1]).

When the nonlinearity is just $\chi^{3}$, a stronger continuous dependence estimate holds. Indeed, we have

Theorem 3.4. Assume that (K1)-(K3) and (H1)-(H2) hold. Let $\left\{f_{i}, \vartheta_{0 i}, \chi_{0 i}, \eta_{0 i}\right\}, i=1,2$, be two sets of data satisfying (H3)-(H6), and let $\left\{\vartheta_{i}, \chi_{i}, \eta_{i}\right\}$ denote the two corresponding solutions to problem $\mathbf{P}$ in the interval $I=[\tau, T]$. Then there exists a positive constant $\Lambda_{2}$ such that, for any $t \in I$,

$$
\begin{aligned}
\| \vartheta_{1}(t) & -\vartheta_{2}(t)\left\|_{H}^{2}+\right\| \chi_{1}(t)-\chi_{2}(t) \|_{V}^{2} \\
& +\int_{\tau}^{t}\left\|\chi_{1}(y)-\chi_{2}(y)\right\|_{W}^{2} d y+\left\|\eta_{1}^{t}-\eta_{2}^{t}\right\|_{\mathcal{M}}^{2} \\
\leq & \Lambda_{2}\left(\left\|\vartheta_{01}-\vartheta_{02}\right\|_{H}^{2}+\left\|\chi_{01}-\chi_{02}\right\|_{V}^{2}\right. \\
& \left.+\left\|\eta_{01}-\eta_{02}\right\|_{\mathcal{M}}^{2}+\left\|f_{1}-f_{2}\right\|_{L^{1}(I, H)+L^{2}\left(I, V^{*}\right)}^{2}\right) .
\end{aligned}
$$

Remark 3.5. A careful look at the proof of Theorem 3.4 (see Section 5 below) shows that a control of $\left\|\vartheta_{1}(t)-\vartheta_{2}(t)\right\|_{H}$ for any $t \in I$ can be obtained even though $\chi^{3}$ is replaced by a maximal monotone graph $\beta$ in $\mathbb{R}^{2}$ (cf. Remark 3.3) provided that $\lambda^{\prime}$ is bounded.

In the sequel, we will denote by $U_{f}(t, \tau) z_{0}$ the solution $(\vartheta, \chi, \eta)$ to problem $\mathbf{P}$ at time $t$ with source term $f$ and initial data $z_{0}=\left(\vartheta_{0}, \chi_{0}, \eta_{0}\right) \in \mathcal{H}$ given at time $\tau$.

Remark 3.6. As an immediate consequence of Theorem 3.2 and Theorem 3.4 , for any fixed $f \in L_{\text {loc }}^{1}(\mathbb{R}, H)+L_{\text {loc }}^{2}\left(\mathbb{R}, V^{*}\right)$, the two-parameter family of operators $U_{f}(t, \tau)$, with $t \geq \tau, \tau \in \mathbb{R}$, satisfies the following properties:

$$
U_{f}(t, \tau): \mathcal{H} \rightarrow \mathcal{H} \text { for any } t \geq \tau, \tau \in \mathbb{R}
$$$$
U_{f}(\tau, \tau) \text { is the identity map on } \mathcal{H} \text { for any } \tau \in \mathbb{R} \text {; }
$$$$
U_{f}(t, s) U_{f}(s, \tau)=U_{f}(t, \tau) \text { for any } t \geq s \geq \tau, \tau \in \mathbb{R} ;
$$$$
U_{f}(t, \tau) z \rightarrow z \text { as } t \downarrow \tau \text { for any } z \in \mathcal{H}, \tau \in \mathbb{R} \text {; }
$$$$
U_{f}(t, \tau) \in C^{0}(\mathcal{H}, \mathcal{H}) \text { for any } \tau \in \mathbb{R}, t \geq \tau \text {. }
$$

Thus, $U_{f}(t, \tau)$ is a (strongly continuous) process with symbol $f$, according to the usual definition (see, e.g., [Har], Chapter 6). 


\section{Proof of Theorem 3.2}

The proof is divided into four subsections. The first is devoted to prove estimate (3.15), which entails uniqueness. The latter are concerned with existence, which is obtained by means of a Faedo-Galerkin approximating scheme (see [BL1] and [GGP]).

4.1. Estimate (3.15). Consider two possible solutions $\left\{\vartheta_{i}, \chi_{i}, \eta_{i}\right\}, i=1,2$, to problem $\mathbf{P}$ corresponding to the source terms and initial data $\left\{f_{i}, \vartheta_{0 i}, \chi_{0 i}, \eta_{0 i}\right\}$. Moreover, set $\tilde{\lambda}=\lambda\left(\chi_{1}\right)-\lambda\left(\chi_{2}\right)$ and (cf. (3.13)-(3.14))

$$
\begin{array}{ll}
\omega=\omega_{1}-\omega_{2}, & \omega_{0}=\omega_{01}-\omega_{02} \\
\chi=\chi_{1}-\chi_{2}, & \chi_{0}=\chi_{01}-\chi_{02} \\
\eta=\eta_{1}-\eta_{2}, & \eta_{0}=\eta_{01}-\eta_{02} .
\end{array}
$$

Then, according to Definition 3.1, $(\omega, \chi, \eta)$ fulfills the system

$$
\begin{aligned}
\left\langle\omega_{t}, v\right\rangle_{V^{*} V}+\langle\omega, v\rangle_{V}=\langle\nabla \tilde{\lambda}, \nabla v\rangle_{H^{3}}-\int_{0}^{\infty} \nu(\sigma)\langle\eta(\sigma), v\rangle_{H} d \sigma \\
-\int_{0}^{\infty} \mu(\sigma)\langle\eta(\sigma), v\rangle_{V} d \sigma+\langle f, v\rangle_{V^{*} V}
\end{aligned}
$$

for all $v \in V$, a.e. in $I$

$$
\begin{aligned}
& \chi_{t}-\Delta \chi+\chi_{1}^{3}-\chi_{2}^{3}= \gamma\left(\chi_{1}\right)-\gamma\left(\chi_{2}\right)+\lambda^{\prime}\left(\chi_{1}\right)\left(\omega_{1}-\lambda\left(\chi_{1}\right)\right) \\
&-\lambda^{\prime}\left(\chi_{2}\right)\left(\omega_{2}-\lambda\left(\chi_{2}\right)\right) \quad \text { a.e. in } \Omega \times I \\
&\left\langle\eta_{t}+\eta_{s}, \psi\right\rangle_{\mathcal{M}}=\langle\omega-\tilde{\lambda}, \psi\rangle_{\mathcal{M}} \quad \text { for all } \psi \in \mathcal{M} \text {, a.e. in } I
\end{aligned}
$$

and initial and boundary conditions (3.9), (3.11)-(3.12), and

$$
\omega(\tau)=\omega_{0} \quad \text { a.e. in } \Omega .
$$

Consider the Riesz map $J=-\Delta+\mathbb{I}$ from $V$ onto $V^{*}$, being $\mathbb{I}$ the identity mapping, and set $v=J^{-1} \omega$ in (4.1). We obtain

$$
\begin{aligned}
\frac{1}{2} \frac{d}{d t}\|\omega\|_{V^{*}}^{2}+\|\omega\|_{V^{*}}^{2}+\|\omega\|_{H}^{2}= & \int_{0}^{\infty} \mu(\sigma)\langle\eta(\sigma), \omega\rangle_{V^{*}} d \sigma-\int_{0}^{\infty} \nu(\sigma)\langle\eta(\sigma), \omega\rangle_{V^{*}} d \sigma \\
& -\int_{0}^{\infty} \mu(\sigma)\langle\eta(\sigma), \omega\rangle_{H} d \sigma+\langle\tilde{\lambda}, \omega\rangle_{H}+\langle f, \omega\rangle_{V^{*}}
\end{aligned}
$$


Recalling (K5) and using Young inequality, from the above relation we deduce

$$
\begin{aligned}
\frac{d}{d t}\|\omega\|_{V^{*}}^{2}+\|\omega\|_{H}^{2} \leq & \left(k_{0}+a_{0}-2\right)\|\omega\|_{V^{*}}^{2}+\|\tilde{\lambda}\|_{H}^{2}+\|\eta\|_{\mathcal{N}}^{2} \\
& -2 \int_{0}^{\infty} \mu(\sigma)\langle\eta(\sigma), \omega\rangle_{H} d \sigma+2\|f\|_{V^{*}}\|\omega\|_{V^{*}}
\end{aligned}
$$

We now multiply (4.2) by $\chi$ and integrate over $\Omega$. We obtain

$$
\begin{aligned}
\frac{1}{2} \frac{d}{d t}\|\chi\|_{H}^{2}=-\| & \nabla \chi \|_{H^{3}}^{2}-\left\langle\chi_{1}^{3}-\chi_{2}^{3}, \chi\right\rangle_{H}+\left\langle\gamma\left(\chi_{1}\right)-\gamma\left(\chi_{2}\right), \chi\right\rangle_{H} \\
& +\left\langle\lambda^{\prime}\left(\chi_{2}\right) \omega, \chi\right\rangle_{H}-\left\langle\lambda^{\prime}\left(\chi_{2}\right) \tilde{\lambda}, \chi\right\rangle_{H} \\
& +\left\langle\left(\lambda^{\prime}\left(\chi_{1}\right)-\lambda^{\prime}\left(\chi_{2}\right)\right)\left(\omega_{1}-\lambda\left(\chi_{1}\right)\right), \chi\right\rangle_{H}
\end{aligned}
$$

Observe at once that

$$
-\left\langle\chi_{1}^{3}-\chi_{2}^{3}, \chi\right\rangle_{H} \leq 0
$$

and (cf. (H1))

$$
\left\langle\gamma\left(\chi_{1}\right)-\gamma\left(\chi_{2}\right), \chi\right\rangle_{H} \leq \Gamma\|\chi\|_{H}^{2}
$$

where $\Gamma=\left\|\gamma^{\prime}\right\|_{L^{\infty}(\mathbb{R})}$. On the other hand, recalling (H2), we can find a positive constant $c$ such that

$$
\begin{aligned}
|\lambda(r)| & \leq c\left(1+r^{2}\right) & & \text { for all } r \in \mathbb{R} \\
\left|\lambda\left(r_{1}\right)-\lambda\left(r_{2}\right)\right| & \leq c\left(1+\left|r_{1}\right|+\left|r_{2}\right|\right)\left|r_{1}-r_{2}\right| & & \text { for all } r_{1}, r_{2} \in \mathbb{R} \\
\left|\lambda^{\prime}(r)\right| & \leq c(1+|r|) & & \text { for all } r \in \mathbb{R} \\
\left|\lambda^{\prime}\left(r_{1}\right)-\lambda^{\prime}\left(r_{2}\right)\right| & \leq c\left|r_{1}-r_{2}\right| & & \text { for all } r_{1}, r_{2} \in \mathbb{R} .
\end{aligned}
$$

Hence, using (4.10)-(4.11) and Young inequality, we get

$$
\begin{aligned}
& \left\langle\lambda^{\prime}\left(\chi_{2}\right) \omega, \chi\right\rangle_{H} \leq \frac{1}{2}\|\omega\|_{H}^{2}+c\left(1+\left\|\chi_{2}\right\|_{C^{0}(\bar{\Omega})}^{2}\right)\|\chi\|_{H}^{2} \\
& \left\langle\lambda^{\prime}\left(\chi_{2}\right) \tilde{\lambda}, \chi\right\rangle_{H} \leq c\left(1+\left\|\chi_{1}\right\|_{C^{0}(\bar{\Omega})}^{2}+\left\|\chi_{2}\right\|_{C^{0}(\bar{\Omega})}^{2}\right)\|\chi\|_{H}^{2}
\end{aligned}
$$


Moreover, note that

$$
\begin{aligned}
& \left\langle\left(\lambda^{\prime}\left(\chi_{1}\right)-\lambda^{\prime}\left(\chi_{2}\right)\right)\left(\omega_{1}-\lambda\left(\chi_{1}\right)\right), \chi\right\rangle_{H} \\
& \quad \leq c\left(\left\|\omega_{1}\right\|_{H}+\left\|\lambda\left(\chi_{1}\right)\right\|_{H}\right)\|\chi\|_{L^{4}(\Omega)}^{2} \\
& \quad \leq c\left(1+\left\|\omega_{1}\right\|_{H}+\left\|\chi_{1}\right\|_{L^{4}(\Omega)}^{2}\right)\|\chi\|_{L^{4}(\Omega)}^{2} \\
& \quad \leq c\left(1+\left\|\omega_{1}\right\|_{H}+\left\|\chi_{1}\right\|_{V}^{2}\right)\|\chi\|_{L^{4}(\Omega)}^{2}
\end{aligned}
$$

where we used (4.9) and (4.12). Then, setting (cf. (3.1), (3.3), and (3.13))

$$
\Lambda_{3}=\sup _{t \in I}\left(1+\left\|\omega_{1}(t)\right\|_{H}+\left\|\chi_{1}(t)\right\|_{V}^{2}\right)
$$

and using Gagliardo-Nirenberg and Young inequalities, we infer from (4.15)

$$
\left\langle\left(\lambda^{\prime}\left(\chi_{1}\right)-\lambda^{\prime}\left(\chi_{2}\right)\right)\left(\omega_{1}-\lambda\left(\chi_{1}\right)\right), \chi\right\rangle_{H} \leq \frac{1}{2}\|\nabla \chi\|_{H^{3}}^{2}+\left(1+c \Lambda_{3}\right)\|\chi\|_{H}^{2} .
$$

On account of the injection $W \hookrightarrow C^{0}(\bar{\Omega})$ and combining (4.7)-(4.8), (4.13)-(4.14), (4.16), with (4.6), we deduce

$$
\frac{d}{d t}\|\chi\|_{H}^{2}+\|\nabla \chi\|_{H^{3}}^{2} \leq \frac{1}{2}\|\omega\|_{H}^{2}+c\left(1+\Lambda_{3}+\zeta\right)\|\chi\|_{H}^{2}
$$

where

$$
\zeta(t)=\left\|\chi_{1}(t)\right\|_{W}^{2}+\left\|\chi_{2}(t)\right\|_{W}^{2} \quad \text { for a.e. } t \in I .
$$

Note that $\zeta \in L^{1}(I)$ due to (3.3). Recalling (4.3), we have, by density,

$$
\left\langle\eta_{t}+\eta_{s}, \psi\right\rangle_{\mathcal{N}}=\langle\omega-\tilde{\lambda}, \psi\rangle_{\mathcal{N}} \quad \text { for all } \psi \in \mathcal{N}, \text { a.e. in } I
$$

Then, we can formally take $\psi=\eta$ and we end up with (see [GGP] for the justification)

(4.20) $\frac{d}{d t}\|\eta\|_{\mathcal{N}}^{2} \leq a_{0}\|\omega\|_{V^{*}}^{2}+\left(k_{0}+a_{0}\right)\|\tilde{\lambda}\|_{H}^{2}+2\|\eta\|_{\mathcal{N}^{*}}^{2}+2 \int_{0}^{\infty} \mu(\sigma)\langle\eta(\sigma), \omega\rangle_{H} d \sigma$. 
Adding together (4.5) and (4.20), we get

$$
\begin{gathered}
\frac{d}{d t}\left(\|\omega\|_{V^{*}}^{2}+\|\eta\|_{\mathcal{N}}^{2}\right)+\|\omega\|_{H}^{2} \leq( \\
\left.k_{0}+2 a_{0}-2\right)\|\omega\|_{V^{*}}^{2}+\left(k_{0}+a_{0}+1\right)\|\tilde{\lambda}\|_{H}^{2} \\
+3\|\eta\|_{\mathcal{N}^{+}}^{2}+2\|f\|_{V^{*}}\|\omega\|_{V^{*}}
\end{gathered}
$$

which yields, thanks to (4.10) and recalling (4.18),

$$
\begin{aligned}
& \frac{d}{d t}\left(\|\omega\|_{V^{*}}^{2}+\|\eta\|_{\mathcal{N}}^{2}\right)+\|\omega\|_{H}^{2} \\
& \quad \leq c\left(\|\omega\|_{V^{*}}^{2}+(1+\zeta)\|\chi\|_{H}^{2}+\|\eta\|_{\mathcal{N}^{*}}^{2}+\|f\|_{V^{*}}\|\omega\|_{V^{*}}\right)
\end{aligned}
$$

Hence, we can combine (4.21) with (4.17). This gives

$$
\frac{d}{d t} \Phi^{2} \leq c\left(\|\omega\|_{V^{*}}^{2}+\left(1+\Lambda_{3}+\zeta\right)\|\chi\|_{H}^{2}+\|\eta\|_{\mathcal{N}^{*}}^{2}+\|f\|_{V^{*}}\|\omega\|_{V^{*}}\right)
$$

where

$$
\Phi^{2}(t)=\|\omega(t)\|_{V^{*}}^{2}+\int_{\tau}^{t}\|\omega(y)\|_{H}^{2} d y+\|\chi(t)\|_{H}^{2}+\int_{\tau}^{t}\|\nabla \chi(y)\|_{H^{3}}^{2} d y+\left\|\eta^{t}\right\|_{\mathcal{N}}^{2}
$$

for any $t \in I$. Finally, from (4.22) we derive

$$
\frac{d}{d t} \Phi^{2}(t) \leq c(1+\zeta(t)) \Phi^{2}(t)+c\|f(t)\|_{V^{*}} \Phi(t) \quad \text { for a.e. } t \in I
$$

and an application of Lemma 2.4 yields (3.15). Of course, we get $\omega=\chi=\eta \equiv 0$ and therefore $\vartheta_{1} \equiv \vartheta_{2}$, whenever the two sets of data coincide.

4.2. Faedo-Galerkin approximation. Let $\left\{v_{j}\right\}_{j=1}^{\infty}$ be a complete set of normalized eigenfunctions of $-\Delta$ in $V$ satisfying Neumann boundary conditions, that is

$$
\begin{aligned}
& -\Delta v_{j}=\alpha_{j} v_{j} \quad \text { in } \Omega \\
& \partial_{\mathbf{n}} v_{j}=0 \quad \text { on } \partial \Omega
\end{aligned}
$$

where $\alpha_{j}$ is the eigenvalue corresponding to $v_{j}$. We recall that $\left\{v_{j}\right\}_{j=1}^{\infty}$ is a smooth orthonormal basis of $H$ which is orthogonal in $V$ as well. Take now a orthonormal basis $\left\{l_{j}\right\}_{j=1}^{\infty} \subset \mathcal{D}\left(\mathbb{R}^{+}\right)$(compactly supported infinitely differentiable 
functions) of $L_{\mu+\nu}^{2}\left(\mathbb{R}^{+}\right)$and consider the set of vectors $\left\{l_{k} v_{h}\right\}_{k, h=1}^{\infty}$. One can easily check that the chosen set forms a orthonormal basis $\left\{z_{j}\right\}_{j=1}^{\infty} \subset \mathcal{D}\left(\mathbb{R}^{+}, V\right)$ of $\mathcal{M}$. For any integer $n$, denote by $P_{n}$ and $Q_{n}$ the projections onto the subspaces

$$
V_{n}=\operatorname{Span}\left\{v_{1}, \ldots, v_{n}\right\} \subset V \quad \text { and } \quad \mathcal{M}_{n}=\operatorname{Span}\left\{z_{1}, \ldots, z_{n}\right\} \subset \mathcal{M}
$$

respectively. Observe that, for any $j$,

$$
v_{j} \in W \quad \text { and } \quad z_{j} \in L_{\nu}^{2}\left(\mathbb{R}^{+}, W\right) \cap L_{\mu}^{2}\left(\mathbb{R}^{+}, W\right) .
$$

It is convenient to approximate $f$ with a sequence $\left\{f_{n}\right\}$ such that

(4.23) $\left\{f_{n}\right\} \subset C^{0}(I, H)$

(4.24) $f_{n}=f_{n}^{1}+f_{n}^{2} \quad$ with $f_{n}^{1} \rightarrow f^{1}$ in $L^{1}(I, H), f_{n}^{2} \rightarrow f^{2}$ in $L^{2}\left(I, V^{*}\right)$

where $\left(f^{1}, f^{2}\right)$ is some fixed decomposition of $f$, that is $f=f^{1}+f^{2}$.

We are now ready to introduce the sequence of approximating problems.

Problem $\boldsymbol{P}_{n}$. Find $t_{n} \in(\tau, T]$ and $a_{j}, b_{j}, c_{j} \in C^{1}\left(\left[\tau, t_{n}\right]\right),(j=1, \ldots, n)$, such that, setting

$$
\vartheta_{n}(t)=\sum_{j=1}^{n} a_{j}(t) v_{j}, \quad \chi_{n}(t)=\sum_{j=1}^{n} b_{j}(t) v_{j}, \quad \eta_{n}^{t}(s)=\sum_{j=1}^{n} c_{j}(t) z_{j}(s)
$$

the triplet $\left(\vartheta_{n}, \chi_{n}, \eta_{n}\right)$ fulfills

$$
\vartheta_{n}, \chi_{n} \in C^{1}\left(\left[\tau, t_{n}\right], W\right)
$$

$$
\eta_{n} \in C^{1}\left(\left[\tau, t_{n}\right], L_{\nu}^{2}\left(\mathbb{R}^{+}, W\right) \cap L_{\mu}^{2}\left(\mathbb{R}^{+}, W\right)\right)
$$

$$
\left\langle\partial_{t}\left(\vartheta_{n}(t)+\lambda\left(\chi_{n}(t)\right)\right), v\right\rangle_{V^{*} V}+\left\langle\nabla \vartheta_{n}(t), v\right\rangle_{V}
$$




$$
\begin{aligned}
+\int_{0}^{\infty} \nu(\sigma)\left\langle\eta_{n}^{t}(\sigma), v\right\rangle_{H} d \sigma+ & \int_{0}^{\infty} \mu(\sigma)\left\langle\nabla \eta_{n}^{t}(\sigma), v\right\rangle_{H^{3}} d \sigma \\
=\left\langle f_{n}(t), v\right\rangle_{V^{*} V} & \text { for all } v \in V_{n}, \text { a.e. in }\left(\tau, t_{n}\right)
\end{aligned}
$$

$$
\left\langle\partial_{t} \chi_{n}, v\right\rangle_{V^{*} V}+\left\langle\nabla \chi_{n}, \nabla v\right\rangle_{H^{3}}+\left\langle\chi_{n}^{3}, v\right\rangle_{H}=\left\langle\gamma\left(\chi_{n}\right)+\lambda^{\prime}\left(\chi_{n}\right) \vartheta_{n}, v\right\rangle_{H}
$$

for all $v \in V_{n}$, a.e. in $\left(\tau, t_{n}\right)$

(4.29) $\left\langle\partial_{t} \eta_{n}+\partial_{s} \eta_{n}, z\right\rangle_{\mathcal{M}}=\left\langle\vartheta_{n}, z\right\rangle_{\mathcal{M}} \quad$ for all $z \in \mathcal{M}_{n}$, a.e. in $\left(\tau, t_{n}\right)$

(4.30) $\vartheta_{n}(\tau)=\vartheta_{0 n}=P_{n} \vartheta_{0}$

a.e. in $\Omega$

(4.31) $\quad \chi_{n}(\tau)=\chi_{0 n}=P_{n} \chi_{0}$

a.e. in $\Omega$

(4.32) $\quad \eta_{n}^{\tau}=\eta_{0 n}=Q_{n} \eta_{0}$

a.e. in $\Omega \times \mathbb{R}^{+}$.

Observe that

$$
\partial_{s} \eta_{n}^{t}(s)= \begin{cases}\vartheta_{n}(t-s) & \text { if } 0<s \leq t-\tau \\ \partial_{s} \eta_{0 n}(s-t+\tau) & \text { if } s>t-\tau\end{cases}
$$

Then, we can easily realize that system (4.27)-(4.29) can be put in normal form. Therefore, an application of a standard fixed-point argument implies that $\mathbf{P}_{n}$ has a (unique) solution with $t_{n}$ small enough.

4.3. A priori estimates. Let us take $v=\vartheta_{n}$ in equation (4.27), $v=\partial_{t} \chi_{n}$ in equation (4.28), and $z=\eta_{n}$ in equation (4.29). Adding the three relations together, we obtain

$$
\begin{gathered}
(4.33) \frac{1}{2} \frac{d}{d t}\left(\left\|\vartheta_{n}\right\|_{H}^{2}+2 \int_{\tau}^{t}\left\|\nabla \vartheta_{n}(y)\right\|_{H^{3}}^{2} d y+\left\|\nabla \chi_{n}\right\|_{H^{3}}^{2}+\left\|\eta_{n}\right\|_{\mathcal{M}^{2}}^{2}+2\left\|\chi_{n}\right\|_{L^{4}(\Omega)}^{4}\right) \\
=-\left\|\vartheta_{n}\right\|_{H}^{2}-\left\|\partial_{t} \chi_{n}\right\|_{H}^{2}+\left\langle f_{n}, \vartheta_{n}\right\rangle_{H}+\left\langle\gamma\left(\chi_{n}\right), \partial_{t} \chi_{n}\right\rangle_{H} \\
\quad+\int_{0}^{\infty} \mu(\sigma)\left\langle\eta_{n}(\sigma), \vartheta_{n}\right\rangle_{H} d \sigma-\left\langle\partial_{s} \eta_{n}, \eta_{n}\right\rangle_{\mathcal{M}}
\end{gathered}
$$

Recall now that (see [GMP1] for details)

$$
\left\langle\partial_{s} \eta_{n}, \eta_{n}\right\rangle_{\mathcal{M}}=-\frac{1}{2} \int_{0}^{\infty} \nu^{\prime}(\sigma)\left\|\eta_{n}(\sigma)\right\|_{H}^{2} d \sigma-\frac{1}{2} \int_{0}^{\infty} \mu^{\prime}(\sigma)\left\|\eta_{n}(\sigma)\right\|_{V}^{2} d \sigma \geq 0
$$

Then, taking (H1), (K5), (4.24), (4.34) into account, and using Young inequality, 
we deduce

$$
\begin{aligned}
(4.35) \frac{1}{2} \frac{d}{d t} & \left(\left\|\vartheta_{n}\right\|_{H}^{2}+\int_{\tau}^{t}\left\|\nabla \vartheta_{n}(y)\right\|_{H^{3}}^{2} d y+\left\|\nabla \chi_{n}\right\|_{H^{3}}^{2}+\left\|\eta_{n}\right\|_{\mathcal{M}}^{2}+2\left\|\chi_{n}\right\|_{L^{4}(\Omega)}^{4}\right) \\
\leq & c\left(1+\left\|\vartheta_{n}\right\|_{H}^{2}+\left\|\chi_{n}\right\|_{V}^{2}+\left\|\eta_{n}\right\|_{\mathcal{M}}^{2}\right)+\left\|f_{n}^{1}\right\|_{H}\left\|\vartheta_{n}\right\|_{H} \\
& +\frac{1}{2}\left\|f_{n}^{2}\right\|_{V^{*}}^{2}-\frac{3}{4}\left\|\partial_{t} \chi_{n}\right\|_{H^{*}}^{2}
\end{aligned}
$$

Hence, adding to (4.35) the elementary inequality

$$
\frac{1}{2} \frac{d}{d t}\left\|\chi_{n}\right\|_{H}^{2} \leq \frac{1}{4}\left\|\partial_{t} \chi_{n}\right\|_{H}^{2}+c\left\|\chi_{n}\right\|_{H}^{2}
$$

we infer

$$
\frac{d}{d t} \Phi^{2} \leq c\left(1+\left\|\vartheta_{n}\right\|_{H}^{2}+\left\|\chi_{n}\right\|_{V}^{2}+\left\|\eta_{n}\right\|_{\mathcal{M}^{2}}^{2}+\left\|f_{n}^{1}\right\|_{H}\left\|\vartheta_{n}\right\|_{H}+\left\|f_{n}^{2}\right\|_{V^{*}}^{2}\right)
$$

where

$$
\begin{aligned}
\Phi^{2}(t)=1 & +\left\|\vartheta_{n}(t)\right\|_{H}^{2}+\int_{\tau}^{t}\left\|\nabla \vartheta_{n}(y)\right\|_{H}^{2} d y+\left\|\chi_{n}(t)\right\|_{V}^{2} \\
& +\left\|\eta_{n}^{t}\right\|_{\mathcal{M}}^{2}+\left\|\chi_{n}(t)\right\|_{L^{4}(\Omega)}^{4}+\int_{\tau}^{t}\left\|\partial_{t} \chi_{n}(y)\right\|_{H}^{2} d y
\end{aligned}
$$

Therefore, from (4.36) we derive the inequality

$$
\frac{d}{d t} \Phi^{2}(t) \leq c \Phi^{2}(t)+c\left\|f_{n}^{1}(t)\right\|_{H} \Phi(t)+c\left\|f_{n}^{2}(t)\right\|_{V^{*}}^{2} \quad \text { for a.e. } t \in I
$$

and applying Lemma 2.4 we get the a priori estimates

$$
\begin{aligned}
\left\|\vartheta_{n}\right\|_{L^{\infty}(I, H) \cap L^{2}(I, V)} & \leq c \\
\left\|\chi_{n}\right\|_{L^{\infty}(I, V) \cap H^{1}(I, H)} & \leq c \\
\left\|\eta_{n}\right\|_{L^{\infty}(I, \mathcal{M})} & \leq c
\end{aligned}
$$

for some positive constant $c$ independent of $n, t_{n}$, and $\lambda$. In particular, the above estimates imply that $t_{n}=T$ for any $n$. Thus, $\left(\vartheta_{n}, \chi_{n}, \eta_{n}\right)$ solves $\mathbf{P}_{n}$ on the whole $I$. 
Let us now take $v=-\Delta \chi_{n}$ in equation (4.28). We have

(4.40) $\frac{1}{2} \frac{d}{d t}\left\|\nabla \chi_{n}\right\|_{H^{3}}^{2}+\left\|\Delta \chi_{n}\right\|_{H}^{2}=-\left\langle 3 \chi_{n}^{2} \nabla \chi_{n}, \nabla \chi_{n}\right\rangle_{H^{3}}-\left\langle\gamma\left(\chi_{n}\right), \Delta \chi_{n}\right\rangle_{H}$

$$
-\left\langle\lambda^{\prime}\left(\chi_{n}\right) \vartheta_{n}, \Delta \chi_{n}\right\rangle_{H} \text {. }
$$

Observe that, using Young and Gagliardo-Nirenberg inequalities and recalling (H1), (4.11), (4.38)-(4.39), we obtain

$$
\begin{aligned}
\left\langle 3 \chi_{n}^{2} \nabla \chi_{n}, \nabla \chi_{n}\right\rangle_{H^{3}} & \geq 0 \\
\left\langle\gamma\left(\chi_{n}\right), \Delta \chi_{n}\right\rangle_{H} & \leq c+\frac{1}{4}\left\|\Delta \chi_{n}\right\|_{H}^{2} \\
\left\langle\lambda^{\prime}\left(\chi_{n}\right) \vartheta_{n}, \Delta \chi_{n}\right\rangle_{H} & \leq c\left(1+\left\|\vartheta_{n}\right\|_{V}^{2}\right)+\frac{1}{4}\left\|\Delta \chi_{n}\right\|_{H}^{2} .
\end{aligned}
$$

Combining (4.40) with (4.38) and (4.41)-(4.43), we find $c>0$ independent of $n$ such that

$$
\left\|\chi_{n}\right\|_{L^{2}(I, W)} \leq c
$$

4.4. Passage to the limit. In force of (4.37)-(4.39) and (4.44), we can find a triplet $(\vartheta, \chi, \eta)$ such that, up to subsequences,

(4.45) $\vartheta_{n} \rightarrow \vartheta \quad$ weakly star in $L^{\infty}(I, H)$, weakly in $L^{2}(I, V)$

(4.46) $\chi_{n} \rightarrow \chi \quad$ weakly star in $L^{\infty}(I, V)$, weakly in $H^{1}(I, H) \cap L^{2}(I, W)$

(4.47) $\eta_{n} \rightarrow \eta \quad$ weakly star in $L^{\infty}(I, \mathcal{M})$.

In addition, on account of (4.46), a classical compactness argument entails

$$
\chi_{n} \rightarrow \chi \text { strongly in } C^{0}(I, H) \cap L^{2}(I, V) .
$$

Recalling (H1), (H2), (4.10), (4.12), and (4.38), owing to (4.48), we have

$$
\begin{aligned}
& \gamma\left(\chi_{n}\right) \rightarrow \gamma(\chi) \quad \text { strongly in } C^{0}(I, H) \\
& \lambda\left(\chi_{n}\right) \rightarrow \lambda(\chi) \quad \text { strongly in } C^{0}(I, H) \\
& \lambda^{\prime}\left(\chi_{n}\right) \rightarrow \lambda^{\prime}(\chi) \text { strongly in } L^{2}\left(I, L^{4}(\Omega)\right) \\
& \chi_{n}^{3} \rightarrow \chi^{3} \quad \text { strongly in } C^{0}(I, H) .
\end{aligned}
$$


Here we also use Hölder inequality and the injection $V \hookrightarrow L^{6}(\Omega)$.

Collecting all the convergences (4.45)-(4.52), and recalling (4.24), we easily realize that $(\vartheta, \chi, \eta)$ fulfills $(3.3),(3.7)-(3.11)$.

In order to get (3.6), let us integrate equation (4.27) with respect to time over $(\tau, t)$. We obtain

$$
\begin{aligned}
& \left\langle\vartheta_{n}, v\right\rangle_{V^{*} V}+\left\langle\lambda\left(\chi_{n}\right), v\right\rangle_{H}+\left\langle\int_{\tau}^{t} \vartheta_{n}(y) d y, v\right\rangle_{V} \\
& +\int_{0}^{\infty} \nu(\sigma)\left\langle\int_{\tau}^{t} \eta_{n}(\sigma, y) d y, v\right\rangle_{H} d \sigma \\
& +\int_{0}^{\infty} \mu(\sigma)\left\langle\nabla \int_{\tau}^{t} \eta_{n}(\sigma, y) d y, \nabla v\right\rangle_{H^{3}} d \sigma \\
& =\left\langle\vartheta_{0 n}+\lambda\left(\chi_{0 n}\right), v\right\rangle_{H}+\left\langle\int_{\tau}^{t} f_{n}(y) d y, v\right\rangle_{V^{*} V} \text { for all } v \in V_{n} \text {, a.e. in } I .
\end{aligned}
$$

Thanks to $(4.24),(4.30)-(4.32),(4.45),(4.46),(4.50)$, we can easily pass to the limit in (4.53). This yields

$$
\begin{gathered}
\langle\vartheta, v\rangle_{V^{*} V}+\langle\lambda(\chi), v\rangle_{H}+\left\langle\int_{\tau}^{t} \vartheta(y) d y, v\right\rangle_{V} \\
+\int_{0}^{\infty} \nu(\sigma)\left\langle\int_{\tau}^{t} \eta(\sigma, y) d y, v\right\rangle_{H} d \sigma \\
+\int_{0}^{\infty} \mu(\sigma)\left\langle\nabla \int_{\tau}^{t} \eta(\sigma, y) d y, \nabla v\right\rangle_{H^{3}} d \sigma \\
=\left\langle\vartheta_{0}+\lambda\left(\chi_{0}\right), v\right\rangle_{H}+\left\langle\int_{\tau}^{t} f(y) d y, v\right\rangle_{V^{*} V} \text { for all } v \in V, \text { a.e. in } I .
\end{gathered}
$$

Thus, from (4.54), we easily recover (3.6) and, by comparison, (3.2), thank to (H3). Consequently, since $\vartheta \in L^{2}(I, V)$, we deduce $\vartheta \in C^{0}(I, H)$ so that (3.1) and (3.5) hold as well. Finally, we have

$$
\eta^{t}(s)= \begin{cases}\int_{t-s}^{t} \vartheta(y) d y & \text { if } 0<s \leq t-\tau \\ \eta_{0}(s-t+\tau)+\int_{\tau}^{t} \vartheta(y) d y & \text { if } s>t-\tau\end{cases}
$$

from which, thanks to (3.1), relations (3.4) and (3.12) follow. 
We conclude observing that, owing to uniqueness, the whole sequence $\left\{\vartheta_{n}, \chi_{n}, \eta_{n}\right\}$ converges to the solution according to (4.45)-(4.52).

\section{Proof of Theorem 3.4}

With the notation of Subsection 4.1, consider equations (4.1)-(4.3). Take $v=\omega$ in equation (4.1), to obtain

$$
\begin{gathered}
\frac{1}{2} \frac{d}{d t}\|\omega\|_{H}^{2}=-\|\omega\|_{H}^{2}-\|\nabla \omega\|_{H}^{2}+\langle\nabla \tilde{\lambda}, \nabla \omega\rangle-\int_{0}^{\infty} \nu(\sigma)\langle\eta(\sigma), \omega\rangle_{H} d \sigma \\
-\int_{0}^{\infty} \mu(\sigma)\langle\nabla \eta(\sigma), \nabla \omega\rangle_{H^{3}} d \sigma-\langle\tilde{\lambda}, \omega\rangle_{H}+\langle f, \omega\rangle
\end{gathered}
$$

Then, taking $\psi=\eta$ in (4.3) we formally deduce

$$
\begin{gathered}
\frac{1}{2} \frac{d}{d t}\|\eta\|_{\mathcal{M}}^{2}=-\left\langle\partial_{s} \eta, \eta\right\rangle_{\mathcal{M}}+\int_{0}^{\infty} \nu(\sigma)\langle\eta(\sigma), \omega\rangle_{H} d \sigma+\int_{0}^{\infty} \mu(\sigma)\langle\eta(\sigma), \omega\rangle_{V} d \sigma \\
-\int_{0}^{\infty} \nu(\sigma)\langle\eta(\sigma), \tilde{\lambda}\rangle_{H} d \sigma-\int_{0}^{\infty} \mu(\sigma)\langle\eta(\sigma), \tilde{\lambda}\rangle_{V} d \sigma
\end{gathered}
$$

To make this argument rigorous, we should perform it in a Faedo-Galerkin scheme (cf. Section 4 and, in particular, (4.29)). Adding (5.1) and (5.2) together, and observing that $\left\langle\partial_{s} \eta, \eta\right\rangle_{\mathcal{M}} \geq 0$ (see (4.34)), we derive the inequality

$$
\begin{aligned}
\frac{1}{2} \frac{d}{d t}\left(\|\omega\|_{H}^{2}+\|\eta\|_{\mathcal{M}}^{2}\right) & \\
\leq & -\|\omega\|_{H}^{2}-\|\nabla \omega\|_{H}^{2}+\langle\nabla \tilde{\lambda}, \nabla \omega\rangle-\int_{0}^{\infty} \nu(\sigma)\langle\eta(\sigma), \omega\rangle_{H} d \sigma \\
& -\int_{0}^{\infty} \mu(\sigma)\langle\nabla \eta(\sigma), \nabla \omega\rangle_{H^{3}} d \sigma-\langle\tilde{\lambda}, \omega\rangle_{H} \\
& +\int_{0}^{\infty} \nu(\sigma)\langle\eta(\sigma), \omega\rangle_{H} d \sigma+\int_{0}^{\infty} \mu(\sigma)\langle\eta(\sigma), \omega\rangle_{V} d \sigma \\
& -\int_{0}^{\infty} \nu(\sigma)\langle\eta(\sigma), \tilde{\lambda}\rangle_{H} d \sigma-\int_{0}^{\infty} \mu(\sigma)\langle\eta(\sigma), \tilde{\lambda}\rangle_{V} d \sigma+\langle f, \omega\rangle .
\end{aligned}
$$


Let us now multiply equation (4.2) by $-\Delta \chi$. We get

$$
\begin{aligned}
\frac{1}{2} \frac{d}{d t} & \|\nabla \chi\|_{H^{3}}^{2}+\|\Delta \chi\|_{H}^{2} \\
= & \left\langle\chi_{1}^{3}-\chi_{2}^{3}, \Delta \chi\right\rangle_{H}-\left\langle\gamma\left(\chi_{1}\right)-\gamma\left(\chi_{2}\right), \Delta \chi\right\rangle_{H} \\
& -\left\langle\lambda^{\prime}\left(\chi_{1}\right)\left(\omega_{1}-\lambda\left(\chi_{1}\right)\right)-\lambda^{\prime}\left(\chi_{2}\right)\left(\omega_{2}-\lambda\left(\chi_{2}\right)\right), \Delta \chi\right\rangle_{H} .
\end{aligned}
$$

Recalling (7.4) in [GGP], we have

$$
\left\|\chi_{1}^{3}-\chi_{2}^{3}\right\|_{H}^{2} \leq c\left(1+\left\|\chi_{1}\right\|_{V}^{4}+\left\|\chi_{2}\right\|_{V}^{4}\right)\|\chi\|_{V}^{2}
$$

and setting

$$
\Lambda_{4}=\sup _{t \in I}\left(1+\left\|\chi_{1}(t)\right\|_{V}^{4}+\left\|\chi_{2}(t)\right\|_{V}^{4}\right)
$$

we deduce

$$
\left\|\chi_{1}^{3}-\chi_{2}^{3}\right\|_{H}^{2} \leq c \Lambda_{4}\|\chi\|_{V}^{2}
$$

Moreover, using (H1), we easily realize that

$$
\left\langle\gamma\left(\chi_{1}\right)-\gamma\left(\chi_{2}\right), \Delta \chi\right\rangle_{H} \leq c\|\chi\|_{H}^{2}+\frac{1}{4}\|\Delta \chi\|_{H}^{2} .
$$

Consider now the identity in $\Omega \times I$

$$
\begin{aligned}
& \lambda^{\prime}\left(\chi_{1}\right)\left(\omega_{1}-\lambda\left(\chi_{1}\right)\right)-\lambda^{\prime}\left(\chi_{2}\right)\left(\omega_{2}-\lambda\left(\chi_{2}\right)\right) \\
& \quad=\lambda^{\prime}\left(\chi_{1}\right) \omega+\left(\lambda^{\prime}\left(\chi_{1}\right)-\lambda^{\prime}\left(\chi_{2}\right)\right) \omega_{2} \\
& \quad+\left(\lambda^{\prime}\left(\chi_{2}\right)-\lambda^{\prime}\left(\chi_{1}\right)\right) \lambda\left(\chi_{1}\right)+\lambda^{\prime}\left(\chi_{2}\right)\left(\lambda\left(\chi_{2}\right)-\lambda\left(\chi_{1}\right)\right) .
\end{aligned}
$$

Observe that (cf. (4.11) and (4.18))

$$
\left\|\lambda^{\prime}\left(\chi_{1}\right) \omega\right\|_{H}^{2} \leq c(1+\zeta)\|\omega\|_{H}^{2} .
$$

Besides, thanks to (4.12), Hölder inequality, and the injection $V \hookrightarrow L^{4}(\Omega)$

$$
\left\|\left(\lambda^{\prime}\left(\chi_{1}\right)-\lambda^{\prime}\left(\chi_{2}\right)\right) \omega_{2}\right\|_{H}^{2} \leq c\left\|\omega_{2}\right\|_{V}^{2}\|\chi\|_{V}^{2}
$$


Then, on account of (4.9)-(4.12), using Hölder inequality, and recalling (4.18) and (5.5), we have

$$
\begin{aligned}
\left\|\left(\lambda^{\prime}\left(\chi_{1}\right)-\lambda^{\prime}\left(\chi_{2}\right)\right) \lambda\left(\chi_{1}\right)\right\|_{H}^{2} & \leq c\left\|\chi_{1}-\chi_{2}\right\|_{L^{6}(\Omega)}^{2}\left(1+\left\|\chi_{1}\right\|_{L^{6}(\Omega)}^{4}\right) \\
& \leq c \Lambda_{4}\left\|\chi_{1}-\chi_{2}\right\|_{V}^{2}
\end{aligned}
$$

and

$$
\begin{aligned}
\left\|\lambda^{\prime}\left(\chi_{2}\right)\left(\lambda\left(\chi_{2}\right)-\lambda\left(\chi_{1}\right)\right)\right\|_{H}^{2} & \leq c(1+\zeta)\left(1+\Lambda_{4}\right)\left\|\chi_{1}-\chi_{2}\right\|_{L^{6}(\Omega)}^{2} \\
& \leq c(1+\zeta)\left(1+\Lambda_{4}\right)\left\|\chi_{1}-\chi_{2}\right\|_{V}^{2}
\end{aligned}
$$

where we have also used the injections $L^{6}(\Omega) \hookrightarrow V$ and $W \hookrightarrow C^{0}(\Omega)$. Consequently, collecting (5.9)-(5.12), from (5.8) we infer

$$
\begin{aligned}
& \left\|\lambda^{\prime}\left(\chi_{1}\right)\left(\omega_{1}-\lambda\left(\chi_{1}\right)\right)-\lambda^{\prime}\left(\chi_{2}\right)\left(\omega_{2}-\lambda\left(\chi_{2}\right)\right)\right\|_{H}^{2} \\
& \quad \leq c\left((1+\zeta)\|\omega\|_{H}^{2}+\left(1+\zeta+\left\|\omega_{2}\right\|_{V}^{2}\right)\|\chi\|_{V}^{2}\right) .
\end{aligned}
$$

Therefore, thanks to (5.13), we have

$$
\begin{aligned}
& \left\langle\lambda^{\prime}\left(\chi_{1}\right)\left(\omega_{1}-\lambda\left(\chi_{1}\right)\right)-\lambda^{\prime}\left(\chi_{2}\right)\left(\omega_{2}-\lambda\left(\chi_{2}\right)\right), \Delta \chi\right\rangle_{H} \\
& \quad \leq c\left((1+\zeta)\|\omega\|_{H}^{2}+\left(1+\zeta+\left\|\omega_{2}\right\|_{V}^{2}\right)\|\chi\|_{V}^{2}\right)+\frac{1}{4}\|\Delta \chi\|_{H}^{2} .
\end{aligned}
$$

Combining now (5.6)-(5.7) with (5.14), from (5.4) we derive the inequality

$$
\frac{d}{d t}\|\nabla \chi\|_{H^{3}}^{2}+\|\Delta \chi\|_{H}^{2} \leq c\left((1+\zeta)\|\omega\|_{H}^{2}+\left(1+\zeta+\left\|\omega_{2}\right\|_{V}^{2}\right)\|\chi\|_{V}^{2}\right)
$$

On the other hand, recalling (4.10) and (5.5), we have

$$
\|\tilde{\lambda}\|_{H}^{2} \leq c \Lambda_{4}\|\chi\|_{V}^{2}
$$

Also, observe that (cf. (H2))

$$
\nabla \tilde{\lambda}=\lambda^{\prime}\left(\chi_{1}\right) \nabla \chi+\left(\lambda^{\prime}\left(\chi_{1}\right)-\lambda^{\prime}\left(\chi_{2}\right)\right) \nabla \chi_{2} \quad \text { a.e. in } \Omega \times I .
$$


Hence, on account of (4.11)-(4.12) and (4.18), we have, by Hölder inequality,

$$
\|\nabla \tilde{\lambda}\|_{H}^{2} \leq c\left(\left\|\chi_{1}\right\|_{C^{0}(\Omega)}^{2}+\left\|\chi_{2}\right\|_{W}^{2}\right)\|\chi\|_{V}^{2} \leq c \zeta\|\chi\|_{V}^{2} .
$$

Consequently, using (5.17) ad Young inequality, we get

$$
\langle\nabla \tilde{\lambda}, \nabla \omega\rangle \leq c \zeta\|\chi\|_{V}^{2}+\frac{1}{4}\|\nabla \omega\|_{H}^{2}
$$

In addition, note that

$$
\langle f, \omega\rangle \leq\left\|f^{1}\right\|_{H}\|\omega\|_{H}+\left\|f^{2}\right\|_{V^{*}}^{2}+\frac{1}{4}\|\omega\|_{V}^{2}
$$

for an arbitrary decomposition $\left(f^{1}, f^{2}\right)$ of $f$ (cf. (H3)). Adding (5.3) to (5.15) and using (5.16), (5.18)-(5.19), we obtain, via Young inequality,

$$
\begin{aligned}
& \frac{d}{d t} \Phi^{2} \leq c\left((1+\zeta)\|\omega\|_{H}^{2}+\left(1+\Lambda_{4}+\zeta+\left\|\omega_{2}\right\|_{V}^{2}\right)\|\chi\|_{V}^{2}+\|\eta\|_{\mathcal{M}}^{2}\right. \\
&\left.+\left\|f^{1}\right\|_{H}\|\omega\|_{H}+\left\|f^{2}\right\|_{V^{*}}^{2}\right)
\end{aligned}
$$

where, for any $t \in I$,

$\Phi^{2}(t)=\|\omega(t)\|_{H}^{2}+\int_{\tau}^{t}\|\nabla \omega(y)\|_{H^{3}}^{2} d y+\|\nabla \chi(t)\|_{H^{3}}^{2}+\int_{\tau}^{t}\|\Delta \chi(y)\|_{H}^{2} d y+\left\|\eta^{t}\right\|_{\mathcal{M}}^{2}$.

Inequality (5.20) yields

$$
\frac{d}{d t} \Phi^{2} \leq c\left(1+\Lambda_{4}+\zeta+\left\|\omega_{2}\right\|_{V}^{2}\right) \Phi^{2}+c\left\|f^{1}\right\|_{H} \Phi+c\left\|f^{2}\right\|_{V^{*}}^{2}
$$

On the other hand, recalling (3.13) and (5.16), we have

$$
\|\vartheta\|_{H}^{2} \leq 2\|\omega\|_{H}^{2}+2 c \Lambda_{4}\|\chi\|_{V}^{2} .
$$

Finally, (3.16) follows applying Lemma 2.4 to (5.21) and taking advantage of (3.15) and (5.22).

\section{Existence of a Uniform Absorbing Set}

The first step towards the existence of a uniform attractor is to prove the existence of a uniform absorbing set. In the sequel, let $\mathcal{F} \subset L_{\text {loc }}^{1}(\mathbb{R}, H)+$ $L_{\text {loc }}^{2}\left(\mathbb{R}, V^{*}\right)$. To get interesting asymptotic properties, we shall require the exponential decays of the kernels $\nu$ and $\mu$, i.e., condition (K4). 
Definition 6.1. $\quad A$ set $\mathcal{B}_{0} \subset \mathcal{H}$ is said to be uniformly absorbing (with respect to $f \in \mathcal{F})$ for the family $\left\{U_{f}(t, \tau), f \in \mathcal{F}\right\}$ if, for any bounded set $\mathcal{B} \subset \mathcal{H}$, there exists $t_{0}=t_{0}(\mathcal{B})$ such that

$$
\bigcup_{f \in \mathcal{F}} U_{f}(t, \tau) \mathcal{B} \subset \mathcal{B}_{0}
$$

for every $\tau \in \mathbb{R}$ and every $t \geq \tau+t_{0}$.

We now state and prove some uniform in time estimates.

Lemma 6.2. Let (K1)-(K4) and (H1)-(H6) hold, and let $f \in \mathcal{T}_{1}+\mathcal{T}_{2}$. Then there exist $\varepsilon>0$ and two continuous increasing functions $C_{j}: \mathbb{R}^{+} \rightarrow \mathbb{R}^{+}$, $j=1$, 2, such that

$$
\left\|U_{f}(t, \tau) z_{0}\right\|_{\mathcal{H}}^{2} \leq C_{1}\left(\left\|z_{0}\right\|_{\mathcal{H}}\right) e^{-\varepsilon(t-\tau)}+C_{2}\left(\|f\|_{\mathcal{T}_{1}+\mathcal{T}_{2}}\right)
$$

for every $t \geq \tau, \tau \in \mathbb{R}$.

Proof. Let $f=f^{1}+f^{2}$ be a fixed decomposition of $f$ such that $\left\|f^{j}\right\|_{\mathcal{T}_{j}} \leq$ $2\|f\|_{\mathcal{T}_{1}+\mathcal{T}_{2}}$. We perform some a priori estimates, which clearly hold in a FaedoGalerkin scheme (see Section 4). Thus, we can proceed formally. Take $v=\vartheta$ in equation (3.6); multiply equation (3.7) by $\chi_{t}$ and then integrate over $\Omega$. Adding the resulting equations, we get

$$
\begin{aligned}
\frac{1}{2} \frac{d}{d t} & \left(\|\vartheta\|_{H}^{2}+\|\nabla \chi\|_{H^{3}}^{2}+\frac{1}{2}\|\chi\|_{L^{4}(\Omega)}^{4}\right)+\|\vartheta\|_{H}^{2}+\|\nabla \vartheta\|_{H^{3}}^{2}+\left\|\chi_{t}\right\|_{H}^{2} \\
= & \left\langle\gamma(\chi), \chi_{t}\right\rangle_{H}+\left\langle f^{1}, \vartheta\right\rangle_{H}+\left\langle f^{2}, \vartheta\right\rangle_{V^{*} V}-\int_{0}^{\infty} \nu(\sigma)\langle\eta(\sigma), \vartheta\rangle_{H} d \sigma \\
& -\int_{0}^{\infty} \mu(\sigma)\langle\nabla \eta(\sigma), \nabla \vartheta\rangle_{H^{3}} d \sigma .
\end{aligned}
$$

Then, multiply equation (3.7) by $\kappa \chi$, for $\kappa>0$, and integrate over $\Omega$, so obtaining

$$
\begin{gathered}
\frac{1}{2} \frac{d}{d t} \kappa\|\chi\|_{H}^{2}+\kappa\|\chi\|_{H}^{2}+\kappa\|\nabla \chi\|_{H^{3}}^{2}+\kappa\|\chi\|_{L^{4}(\Omega)}^{4} \\
=\kappa\langle\tilde{\gamma}(\chi), \chi\rangle_{H}+\kappa\left\langle\lambda^{\prime}(\chi) \vartheta, \chi\right\rangle_{H}
\end{gathered}
$$

where $\tilde{\gamma}(r)=r+\gamma(r)$. Notice that, by $(\mathrm{H} 1), \tilde{\gamma}^{\prime} \in L^{\infty}(\mathbb{R})$. Besides, define

$$
\rho(\eta)=\int_{0}^{\infty} \nu(\sigma)\|\eta(\sigma)\|_{H}^{2} d \sigma+\int_{0}^{\infty} \mu(\sigma)\|\nabla \eta(\sigma)\|_{H^{3}}^{2} d \sigma
$$


Multiply equation (3.8) (considered in the strong sense, i.e., almost everywhere in $I \times \Omega \times \mathbb{R}^{+}$) by $\nu \eta$, then take the gradient of (3.8) and multiply it by $\mu \nabla \eta$; add the resulting equations and integrate on $\Omega \times \mathbb{R}^{+}$. This procedure yields

$$
\begin{aligned}
\frac{1}{2} \frac{d}{d t} \rho(\eta)=- & \frac{1}{2} \int_{0}^{\infty} \nu(\sigma) \frac{d}{d \sigma}\|\eta(\sigma)\|_{H}^{2} d \sigma-\frac{1}{2} \int_{0}^{\infty} \mu(\sigma) \frac{d}{d \sigma}\|\nabla \eta(\sigma)\|_{H^{3}}^{2} d \sigma \\
& +\int_{0}^{\infty} \nu(\sigma)\langle\vartheta, \eta(\sigma)\rangle_{H} d \sigma+\int_{0}^{\infty} \mu(\sigma)\langle\nabla \vartheta, \nabla \eta(\sigma)\rangle_{H^{3}} d \sigma
\end{aligned}
$$

Using (K4) and performing an integration by parts, we have that

$$
\int_{0}^{\infty} \nu(\sigma) \frac{d}{d \sigma}\|\eta(\sigma)\|_{H}^{2} d \sigma+\int_{0}^{\infty} \mu(\sigma) \frac{d}{d \sigma}\|\nabla \eta(\sigma)\|_{H^{3}}^{2} d \sigma \geq \delta \rho(\eta) .
$$

Set

$$
\Phi^{2}(t)=\|\vartheta(t)\|_{H}^{2}+\kappa\|\chi(t)\|_{H}^{2}+\|\nabla \chi(t)\|_{H^{3}}^{2}+\frac{1}{2}\|\chi(t)\|_{L^{4}(\Omega)}^{4}+\rho\left(\eta^{t}\right)
$$

and let $\varepsilon(\kappa)=\min \{1,2 \kappa, \delta\}$. Addition of (6.1)-(6.2) and (6.4), with the help of (6.5), leads to

$$
\begin{aligned}
\frac{d}{d t} \Phi^{2} & +\varepsilon(\kappa) \Phi^{2}+\|\vartheta\|_{H}^{2}+2\|\nabla \vartheta\|_{H^{3}}^{2}+2\left\|\chi_{t}\right\|_{H}^{2} \\
\leq & 2\left\langle\gamma(\chi), \chi_{t}\right\rangle_{H}+2 \kappa\langle\tilde{\gamma}(\chi), \chi\rangle_{H}+2 \kappa\left\langle\lambda^{\prime}(\chi) \vartheta, \chi\right\rangle_{H} \\
& +2\left\langle f^{1}, \vartheta\right\rangle_{H}+2\left\langle f^{2}, \vartheta\right\rangle_{V^{*} V} .
\end{aligned}
$$

By (H1)-(H2) and Young inequality,

$$
2\left\langle\gamma(\chi), \chi_{t}\right\rangle_{H}+2 \kappa\langle\tilde{\gamma}(\chi), \chi\rangle_{H} \leq\left\|\chi_{t}\right\|_{H}^{2}+c+c\|\chi\|_{L^{4}(\Omega)}^{2} \leq\left\|\chi_{t}\right\|_{H}^{2}+c+c \Phi
$$

and

$$
2 \kappa\left\langle\lambda^{\prime}(\chi) \vartheta, \chi\right\rangle_{H} \leq \kappa c\|\vartheta\|_{H}^{2}+\frac{\varepsilon(\kappa)}{4}\|\chi\|_{L^{4}(\Omega)}^{4} \leq \kappa c\|\vartheta\|_{H}^{2}+\frac{\varepsilon(\kappa)}{2} \Phi^{2}
$$

Then choose $\kappa$ small enough so that $\kappa c \leq \frac{1}{2}$. Since

$$
2\left\langle f^{1}, \vartheta\right\rangle_{H} \leq 2\left\|f^{1}\right\|_{H}\|\vartheta\|_{H} \leq 2\left\|f^{1}\right\|_{H} \Phi
$$


and

$$
2\left\langle f^{2}, \vartheta\right\rangle_{V^{*} V} \leq 2\left\|f^{2}\right\|_{V^{*}}\|\vartheta\|_{V} \leq 2\left\|f^{2}\right\|_{V^{*}}^{2}+\frac{1}{2}\|\vartheta\|_{V}^{2}
$$

setting $\varepsilon=\varepsilon(\kappa) / 4$, from (6.6) we conclude that

$$
\frac{d}{d t} \Phi^{2}+2 \varepsilon \Phi^{2}+\|\nabla \vartheta\|_{H^{3}}^{2}+\left\|\chi_{t}\right\|_{H}^{2} \leq c+2\left\|f^{2}\right\|_{V^{*}}^{2}+\left(c+2\left\|f^{1}\right\|_{H}\right) \Phi
$$

and, in particular,

$$
\frac{d}{d t} \Phi^{2}+2 \varepsilon \Phi^{2} \leq c+2\left\|f^{2}\right\|_{V^{*}}^{2}+\left(c+2\left\|f^{1}\right\|_{H}\right) \Phi .
$$

Through the end of the proof, let $C_{1}$ and $C_{2}$ be two generic continuous, positive, increasing functions of $\left\|z_{0}\right\|_{\mathcal{H}}$ and $\|f\|_{\mathcal{T}_{1}+\mathcal{T}_{2}}$, respectively. Exploiting Lemma 2.5, we get

$$
\Phi^{2}(t) \leq 2 \Phi^{2}(\tau) e^{-2 \varepsilon(t-\tau)}+C_{2}
$$

for any $t \in[\tau,+\infty)$. Finally, define

$$
\Psi^{2}(t)=\|\vartheta(t)\|_{H}^{2}+\kappa\|\chi(t)\|_{H}^{2}+\|\nabla \chi(t)\|_{H^{3}}^{2}+\frac{1}{2}\|\chi(t)\|_{L^{4}(\Omega)}^{4}+\left\|\eta^{t}\right\|_{\mathcal{M}}^{2}
$$

Notice that, for every $t \geq \tau, \Phi(t) \leq \Psi(t)$, and

$$
\frac{1}{c} \Psi(t) \leq\left\|U_{f}(t, \tau) z_{0}\right\|_{\mathcal{H}} \leq c \Psi(t) \quad \text { for some } c>1 .
$$

Repeat then the same arguments leading to (6.8), the only difference being that we have to consider (3.8) with $\eta$ in place of $\psi$, instead of (6.4). The result is the following inequality, similar to (6.8),

$$
\frac{d}{d t} \Psi^{2}+2 \varepsilon \Psi^{2} \leq c+2\left\|f^{2}\right\|_{V^{*}}^{2}+\left(c+2\left\|f^{1}\right\|_{H}\right) \Psi+2 \int_{0}^{\infty} \mu(\sigma)\langle\eta(\sigma), \vartheta\rangle_{H}
$$

On the other hand, Young inequality yields

$$
2 \int_{0}^{\infty} \mu(\sigma)\langle\eta(\sigma), \vartheta\rangle_{H} d \sigma \leq \varepsilon\|\eta\|_{\mathcal{M}}^{2}+c\|\vartheta\|_{H}^{2} \leq \varepsilon \Psi^{2}+c \Phi^{2}
$$

and therefore (6.10) turns into

$$
\frac{d}{d t} \Psi^{2}+\varepsilon \Psi^{2} \leq c+2\left\|f^{2}\right\|_{V^{*}}^{2}+\left(c+2\left\|f^{1}\right\|_{H}\right) \Psi+c \Phi^{2}
$$


Observe that (6.9) reads

$$
\Phi^{2}(t) \leq C_{1} e^{-2 \varepsilon(t-\tau)}+C_{2}
$$

and

$$
\int_{\tau}^{t} e^{-2 \varepsilon(y-\tau)} e^{-\varepsilon(t-y)} d y \leq \frac{1}{\varepsilon} e^{-\varepsilon(t-\tau)} .
$$

Thus, a further application of Lemma 2.5 to (6.11) entails

$$
\left\|U_{f}(t, \tau) z_{0}\right\|_{\mathcal{H}}^{2} \leq C_{1} e^{-\varepsilon(t-\tau)}+C_{2}
$$

for any $t \in[\tau,+\infty)$.

Lemma 6.3. Let (K1)-(K4) and (H1)-(H6) hold, and let $f \in \mathcal{T}_{1}$. Then there exists a continuous function $C_{3}: \mathbb{R}^{+} \times \mathbb{R}^{+} \rightarrow \mathbb{R}^{+}$, increasing in both variables, such that

$$
\sup _{\tau \in \mathbb{R}} \sup _{t \geq \tau} \int_{t}^{t+1}\left(\|\nabla \vartheta(y)\|_{H^{3}}^{2}+\left\|\chi_{t}(y)\right\|_{H}^{2}\right) d y \leq C_{3}\left(\left\|z_{0}\right\|_{\mathcal{H}},\|f\|_{\mathcal{T}_{1}}\right)
$$

where $\vartheta(y)$ and $\chi(y)$ are the first and the second component, respectively, of $U_{f}(y, \tau) z_{0}$.

Proof. With reference to Lemma 6.2, integrate (6.7) over $[t, t+1]$, for $t \geq \tau$, to get

$$
\begin{aligned}
& \int_{t}^{t+1}\left(\|\nabla \vartheta(y)\|_{H^{3}}^{2}+\left\|\chi_{t}(y)\right\|_{H}^{2}\right) d y \\
& \quad \leq \Phi^{2}(t)+c+c \int_{t}^{t+1} \Phi(y) d y+2 \int_{t}^{t+1}\|f(y)\|_{H} \Phi(y) d y .
\end{aligned}
$$

In virtue of $(6.12), \Phi^{2}(t) \leq C_{1}+C_{2}$ for every $t \geq \tau$. Hence (6.13) bears

$$
\begin{aligned}
& \int_{t}^{t+1}\left(\|\nabla \vartheta(y)\|_{H^{3}}^{2}+\left\|\chi_{t}(y)\right\|_{H}^{2}\right) d y \\
& \quad \leq C_{1}+C_{2}+c+c\left(C_{1}+C_{2}\right)^{1 / 2}+2\left(C_{1}+C_{2}\right)^{1 / 2}\|f\|_{\mathcal{T}_{1}}
\end{aligned}
$$

as desired.

An immediate consequence of Lemma 6.2 is the existence of a uniform absorbing set, when $f$ is allowed to move in a bounded subset of $\mathcal{T}_{1}+\mathcal{T}_{2}$. 
Theorem 6.4. Let (K1)-(K4) and (H1)-(H6) hold, and let $\mathcal{F} \subset \mathcal{T}_{1}+\mathcal{T}_{2}$ be a bounded set. Denote $M_{\mathcal{F}}=\sup _{f \in \mathcal{F}}\|f\|_{\mathcal{T}_{1}+T_{2}}$. For every $\rho>0$, the ball of $\mathcal{H}$ of radius $C_{2}\left(M_{\mathcal{F}}\right)+\rho$ is a uniform absorbing set for the family $\left\{U_{f}(t, \tau), f \in \mathcal{F}\right\}$.

Remark 6.5. Theorem 6.4 can still be proved when $\chi^{3}$ is replaced by a more general monotone nonlinearity (see $[\mathrm{GGP}]$ ). It is also worth noting that a non-homogeneous Neumann boundary conditions for the temperature can be handled since $f$ may have a component in $\mathcal{T}_{2}$.

The following lemma will be crucial in the course of the investigation.

Lemma 6.6. Let (K1)-(K4) and (H1)-(H6) hold, and let $f \in \mathcal{T}_{1}$. Then there exists a continuous function $C_{4}: \mathbb{R}^{+} \rightarrow \mathbb{R}^{+}$such that

$$
\sup _{\tau \in \mathbb{R}} \sup _{t \geq \tau+1}\left(\left\|\chi_{t}(t)\right\|_{H}+\|\chi(t)\|_{W}\right) \leq C_{4}(R)
$$

whenever $\left\|z_{0}\right\|_{\mathcal{H}} \leq R$ and $\|f\|_{\mathcal{T}_{1}} \leq R$, where $\chi(y)$ is the second component of $U_{f}(y, \tau) z_{0}$.

Proof. Along this proof, the generic constant $c$ will depend on $R$. Take the inner product in $H$ of equation (3.7) with $\chi, \chi_{t},-\Delta \chi$, and $-\Delta \chi_{t}$, respectively. Adding the results we get

$$
\frac{d}{d t}\|\chi\|_{W}^{2}+\left\|\chi_{t}\right\|_{H}^{2}+\|\Delta \chi\|_{H}^{2}+2\|\nabla \chi\|_{H^{3}}^{2}+2\left\|\nabla \chi_{t}\right\|_{H^{3}}^{2}=\sum_{j=1}^{4} I_{j}
$$

where

$$
\begin{aligned}
I_{1}= & -2\left\langle\chi^{3}, \chi\right\rangle_{H}+2\langle\gamma(\chi), \chi\rangle_{H}+2\left\langle\lambda^{\prime}(\chi) \vartheta, \chi\right\rangle_{H} \\
I_{2}=- & \left\langle\chi^{3}, \chi_{t}\right\rangle_{H}+\left\langle\gamma(\chi), \chi_{t}\right\rangle_{H}+\left\langle\lambda^{\prime}(\chi) \vartheta, \chi_{t}\right\rangle_{H} \\
I_{3}= & \left\langle\chi^{3}, \Delta \chi\right\rangle_{H}-\langle\gamma(\chi), \Delta \chi\rangle_{H}-\left\langle\lambda^{\prime}(\chi) \vartheta, \Delta \chi\right\rangle_{H} \\
I_{4}=- & 6\left\langle\chi^{2} \nabla \chi, \nabla \chi_{t}\right\rangle_{H^{3}}+2\left\langle\gamma^{\prime}(\chi) \nabla \chi, \nabla \chi_{t}\right\rangle_{H^{3}} \\
& +2\left\langle\lambda^{\prime \prime}(\chi) \nabla \chi \vartheta, \nabla \chi_{t}\right\rangle_{H^{3}}+2\left\langle\lambda^{\prime}(\chi) \nabla \vartheta, \nabla \chi_{t}\right\rangle_{H^{3}} .
\end{aligned}
$$

To control $I_{j}$, we have to use Lemma 6.2, the generalized Hölder inequality, Young inequality, and the usual embeddings (in particular, $V \hookrightarrow L^{6}(\Omega)$ and $\left.W \hookrightarrow C^{0}(\bar{\Omega})\right)$. Notice first that, given $u \in H$, and $\rho>0$

$$
\left\langle\lambda^{\prime}(\chi) \vartheta, u\right\rangle_{H} \leq\left\|\lambda^{\prime}(\chi)\right\|_{L^{3}(\Omega)}\|\vartheta\|_{L^{6}(\Omega)}\|u\|_{H} \leq c\|\vartheta\|_{V}\|u\|_{H} \leq c\|\vartheta\|_{V}^{2}+\rho\|u\|_{H}^{2} .
$$


Thus it is clear that

$$
I_{1}+I_{2}+I_{3} \leq c+c\|\vartheta\|_{V}^{2}+\left\|\chi_{t}\right\|_{H}^{2}+\|\Delta \chi\|_{H}^{2} .
$$

Concerning $I_{4}$, we have

$$
\begin{aligned}
-6\left\langle\chi^{2} \nabla \chi, \nabla \chi_{t}\right\rangle_{H^{3}} & \leq c\|\chi\|_{V}^{2}\|\chi\|_{W}\left\|\nabla \chi_{t}\right\|_{H^{3}} \leq c\|\chi\|_{W}^{2}+\frac{1}{2}\left\|\nabla \chi_{t}\right\|_{H^{3}}^{2} \\
2\left\langle\gamma^{\prime}(\chi) \nabla \chi, \nabla \chi_{t}\right\rangle_{H^{3}} & \leq c+\frac{1}{2}\left\|\nabla \chi_{t}\right\|_{H^{3}}^{2} \\
2\left\langle\lambda^{\prime \prime}(\chi) \nabla \chi \vartheta, \nabla \chi_{t}\right\rangle_{H^{3}} & \leq c\|\chi\|_{W}\|\vartheta\|_{V}\left\|\nabla \chi_{t}\right\|_{H^{3}} \leq c\|\chi\|_{W}^{2}\|\vartheta\|_{V}^{2}+\frac{1}{2}\left\|\nabla \chi_{t}\right\|_{H^{3}}^{2}
\end{aligned}
$$

and

$$
\begin{aligned}
2\left\langle\lambda^{\prime}(\chi) \nabla \vartheta, \nabla \chi_{t}\right\rangle_{H^{3}} & \leq\left\|\lambda^{\prime}(\chi)\right\|_{L^{\infty}(\Omega)}\|\vartheta\|_{V}\left\|\nabla \chi_{t}\right\|_{H^{3}} \\
& \leq c\left(1+\|\chi\|_{W}\right)\|\vartheta\|_{V}\left\|\nabla \chi_{t}\right\|_{H^{3}} \\
& \leq c\|\vartheta\|_{V}^{2}+c\|\chi\|_{W}^{2}\|\vartheta\|_{V}^{2}+\frac{1}{2}\left\|\nabla \chi_{t}\right\|_{H^{3}}^{2}
\end{aligned}
$$

Thus

$$
\sum_{j=1}^{4} I_{j} \leq\left\|\chi_{t}\right\|_{H}^{2}+\|\Delta \chi\|_{H}^{2}+2\left\|\nabla \chi_{t}\right\|_{H^{3}}^{2}+c+c\|\chi\|_{W}^{2}+c\|\vartheta\|_{V}^{2}+c\|\chi\|_{W}^{2}\|\vartheta\|_{V}^{2}
$$

and we conclude that

$$
\frac{d}{d t}\|\chi\|_{W}^{2} \leq c\left(1+\|\vartheta\|_{V}^{2}\right)\|\chi\|_{W}^{2}+c\left(1+\|\vartheta\|_{V}^{2}\right)
$$

Since

$$
\Delta \chi=\chi_{t}+\chi^{3}-\gamma(\chi)-\lambda^{\prime}(\chi) \vartheta \quad \text { a.e. in } \Omega \times(\tau,+\infty)
$$

in virtue of Lemma 6.2 and Lemma 6.3 we see that, for every $\tau \in \mathbb{R}$ and every $t \geq \tau$, there exist two positive constants $c_{1}$ and $c_{2}$ (depending only on $R$ ), such that

$$
\int_{t}^{t+1}\|\chi(y)\|_{W}^{2} d y \leq c_{1} \text { and } \int_{t}^{t+1}\left(1+\|\vartheta(y)\|_{V}^{2}\right) d y \leq c_{2} .
$$

The result follows then from Lemma 2.3, using again (6.14). 


\section{Existence of a Uniform Attractor}

In the sequel we consider a translation compact function $g$ in $L_{\mathrm{loc}}^{1}(\mathbb{R}, H)$, and we study the asymptotic behavior of the family of processes $U_{f}(t, \tau)$ as $f \in \mathrm{H}(g)$. We first recall some basic definitions. The reader is referred to [BV,Tem] and [Har] for a detailed presentation of the theory of attractors for autonomous and non-autonomous systems, respectively.

Definition 7.1. $A$ set $\mathcal{K} \subset \mathcal{H}$ is said to be uniformly attracting for the family $\left\{U_{f}(t, \tau), f \in \mathrm{H}(g)\right\}$ if, for any $\tau \in \mathbb{R}$ and any bounded set $\mathcal{B} \subset \mathcal{H}$,

$$
\lim _{t \rightarrow \infty}\left[\sup _{f \in \mathrm{H}(g)} \operatorname{dist}\left(U_{f}(t, \tau) \mathcal{B}, \mathcal{K}\right)\right]=0
$$

where

$$
\operatorname{dist}\left(\mathcal{B}_{1}, \mathcal{B}_{2}\right)=\sup _{z_{1} \in \mathcal{B}_{1}} \inf _{z_{2} \in \mathcal{B}_{2}}\left\|z_{1}-z_{2}\right\|_{\mathcal{H}}
$$

denotes the semidistance of two sets $\mathcal{B}_{1}$ and $\mathcal{B}_{2}$ in $\mathcal{H}$. A family of processes that possesses a uniformly attracting compact set is said to be uniformly asymptotically compact.

Definition 7.2. A closed set $\mathcal{A} \subset \mathcal{H}$ is said to be a uniform attractor for the family $\left\{U_{f}(t, \tau), f \in \mathrm{H}(g)\right\}$ if it is at the same time uniformly attracting and contained in every closed uniformly attracting set.

As a direct consequence of the above definition, the uniform attractor of a family of processes (if it exists) is unique.

We shall exploit the following result from [CV1, CV2] (see also the monograph [Vis]).

Theorem 7.3. Let $g$ be a translation compact function in $L_{\text {loc }}^{1}(\mathbb{R}, H)$, and assume that $U_{\bullet}(t, \tau)$ is continuous as a map $\mathcal{H} \times \mathrm{H}(g) \rightarrow \mathcal{H}$, for every $\tau \in \mathbb{R}$ and $t \geq \tau$. If the family $\left\{U_{f}(t, \tau), f \in \mathrm{H}(g)\right\}$ is uniformly asymptotically compact, then it possesses a compact uniform attractor given by

$$
\mathcal{A}=\mid \begin{aligned}
& z(0) \text { such that } z(t) \text { is any bounded complete tra- } \\
& \text { jectory of } U_{f}(t, \tau) \text { for some } f \in \mathrm{H}(g) .
\end{aligned}
$$

It is easy to see that if the system is autonomous, i.e., $f \in H$ independent of time, then $U_{f}(t, 0)$ is a semigroup, and $\mathcal{A}$ is the global attractor of $U_{f}(t, 0)$ on $\mathcal{H}$. Notice that in this case $\mathrm{H}(g)=\{g\}$. We recall that the global attractor is the (unique) compact attracting set which is fully invariant for the semigroup.

For later convenience, we introduce the triplets

$$
z_{0}=\left(\vartheta_{0}, \chi_{0}, \eta_{0}\right) \quad \text { and } \quad z(t)=U_{f}(t, \tau) z_{0}=\left(\vartheta(t), \chi(t), \eta^{t}\right)
$$


Then we can rewrite problem $\mathbf{P}$ in the compact form

$$
\begin{aligned}
z_{t}= & L z+N\left(z, z_{t}\right)+F(t), \quad z(\tau)=z_{0} \\
& + \text { boundary conditions. }
\end{aligned}
$$

The linear operator $L$ has the (formal) expression

$$
L=\left(\begin{array}{ccc}
-\mathbb{I}+\Delta & 0 & -\int_{0}^{\infty} \nu(\sigma) \mathbb{I} \cdot d \sigma+\int_{0}^{\infty} \mu(\sigma) \Delta \cdot d \sigma \\
0 & -\mathbb{I}+\Delta & 0 \\
\mathbb{I} & 0 & -\partial_{s}
\end{array}\right)
$$

and $N$ and $F$ are given, respectively, by

$$
N\left(z, z_{t}\right)=\left(-\lambda^{\prime}(\chi) \chi_{t},-\chi^{3}+\tilde{\gamma}(\chi)+\lambda^{\prime}(\chi) \vartheta, 0\right)
$$

and

$$
F(t)=(f(t), 0,0),
$$

where $\tilde{\gamma}(r)=r+\gamma(r)$. Again, from $(\mathrm{H} 1), \tilde{\gamma}^{\prime} \in L^{\infty}(\mathbb{R})$. as

In view of applying Theorem 7.3 , we write the solution $z=(\vartheta, \chi, \eta)$ to $(7.3)$

$$
z=z_{L}+z_{E}+z_{N}
$$

with $z_{L}=\left(\vartheta_{L}, \chi_{L}, \eta_{L}\right), z_{E}=\left(\vartheta_{E}, \chi_{E}, \eta_{E}\right)$, and $z_{N}=\left(\vartheta_{N}, \chi_{N}, \eta_{N}\right)$, where, neglecting the boundary conditions for the sake of simplicity,

$$
\begin{aligned}
\partial_{t} z_{L} & =L z_{L}, & z_{L}(\tau) & =z_{0}, \\
\partial_{t} z_{E} & =L z_{E}+F(t), & z_{E}(\tau) & =0, \\
\partial_{t} z_{N} & =L z_{N}+N\left(z, z_{t}\right), & z_{N}(\tau) & =0 .
\end{aligned}
$$

It is now apparent to check, along the lines of Theorem 3.2, that systems (7.4)(7.6) admit unique solutions which belong to the space $C^{0}(\mathcal{H}, \mathcal{H})$. In particular, being (7.4) a linear system, there exists a strongly continuous semigroup $S(t)$ of bounded linear operators on $\mathcal{H}$ such that

$$
z_{L}(t)=S(t-\tau) z_{0}
$$

The following lemmas show some basic properties of $z_{L}, z_{E}$, and $z_{N}$. 
Lemma 7.4. The semigroup $S(t)$ has an exponential decay, that is,

$$
\left\|S(t) z_{0}\right\|_{\mathcal{H}} \leq M e^{-\varepsilon_{0} t}\left\|z_{0}\right\|_{\mathcal{H}} \quad \text { for all } z_{0} \in \mathcal{H}
$$

for some $M \geq 1$ and $\varepsilon_{0}>0$.

Proof. Notice first that the second equation of system (7.4) reads

$$
\partial_{t} \chi_{L}=\Delta \chi_{L}-\chi_{L} \quad \text { a.e. in } \Omega \times(\tau,+\infty)
$$

and it is well known that the solution to the above parabolic equation has an exponential decay in $V$. Let then $T(t)$ be the semigroup associated to the first and the third equation of system (7.4) acting on $H \times \mathcal{M}$, namely, $\left(\vartheta_{L}(t), \eta_{L}^{t}\right)=$ $T(t-\tau)\left(\vartheta_{0}, \eta_{0}\right)$. We are left to show that $T(t)$ has an exponential decay. Since the system is linear, there is no harm to assume $\tau=0$. Denote

$$
\Phi^{2}(t)=\left\|\vartheta_{L}(t)\right\|_{H}^{2}+\rho\left(\eta_{L}^{t}\right)
$$

and

$$
\Psi^{2}(t)=\left\|T(t)\left(\vartheta_{0}, \eta_{0}\right)\right\|_{H \times \mathcal{M}}^{2}=\left\|\vartheta_{L}(t)\right\|_{H}^{2}+\left\|\eta_{L}^{t}\right\|_{\mathcal{M}}^{2}
$$

with $\rho$ given by (6.3). Recasting the argument used in Lemma 6.2 (here we do not have the nonlinear term and the source term), we find the inequalities

$$
\frac{d}{d t} \Phi^{2}+2 \varepsilon_{0} \Phi^{2}+2\left\|\nabla \vartheta_{L}\right\|_{H^{3}}^{2} \leq 0
$$

and

$$
\frac{d}{d t} \Psi^{2}+\varepsilon_{0} \Psi^{2} \leq c \Phi^{2}
$$

with $\varepsilon_{0}=\min \{1, \delta\}$. Applying Gronwall lemma to (7.8), inequality (7.9) becomes

$$
\frac{d}{d t} \Psi^{2}(t)+\varepsilon_{0} \Psi^{2}(t) \leq c \Phi^{2}(0) e^{-2 \varepsilon_{0} t} \leq c \Psi^{2}(0) e^{-2 \varepsilon_{0} t}
$$

A further application of Gronwall lemma to (7.10) leads to

$$
\Psi^{2}(t) \leq\left(1+\frac{c}{\varepsilon_{0}}\right) \Psi^{2}(0) e^{-\varepsilon_{0} t}
$$

for any $t \in \mathbb{R}^{+}$, which yields the required exponential decay. 
We now turn our attention to (7.5). In force of (7.7), the solution $z_{E}$ is given by the Duhamel integral

$$
z_{E}(t)=\int_{\tau}^{t} S(t-y)(f(y), 0,0) d y=\int_{0}^{t-\tau} S(t-\tau-y)\left(f^{\tau}(y), 0,0\right) d y
$$

for any $t \geq \tau$. We have

Lemma 7.5. There exists a compact set $\mathcal{K}_{E} \subset \mathcal{H}$ such that

$$
\bigcup_{f \in \mathrm{H}(g)} \bigcup_{\tau \in \mathbb{R}} \bigcup_{t \geq \tau} z_{E}(t) \subset \mathcal{K}_{E}
$$

Proof. Introduce

$$
\mathcal{C}_{E}=\left\{\int_{0}^{t} S(t-y)(f(y), 0,0) d y, t \in \mathbb{R}^{+}, f \in \mathrm{H}(g)\right\}
$$

It is clear from (7.12) that $z_{E}(t) \in \mathcal{C}_{E}$, whenever $f \in \mathrm{H}(g), \tau \in \mathbb{R}$, and $t \geq \tau$. Repeating the argument used in the proof of Lemma 4.3 in [GP] (see also [CV1]), one shows that $\mathcal{C}_{E}$ is relatively compact in $\mathcal{H}$. Hence, let $\mathcal{K}_{E}$ be the closure of $\mathcal{C}_{E}$ in $\mathcal{H}$.

Finally, we analyze the solution $z_{N}$ to (7.6). In the sequel, let $\mathcal{B}_{0}$ be a uniform absorbing set for the family $\left\{U_{f}(t, \tau), f \in \mathrm{H}(g)\right\}$ (which exists by Theorem $6.4)$, and denote $R_{0}=\sup _{z_{0} \in \mathcal{B}_{0}}\left\|z_{0}\right\|_{\mathcal{H}}$.

Lemma 7.6. Assume that $z_{0} \in \mathcal{B}_{0}$. Then there exists a constant $K$ (depending only on $R_{0}$ and $\|g\|_{\mathcal{T}_{1}}$ ) such that

$$
\left\|z_{N}(t)\right\|_{\mathcal{V}} \leq K
$$

for every $\tau \in \mathbb{R}$ and every $t \geq \tau+1$.

Proof. It is convenient to write down (7.6) explicitly, namely,

(7.14) $\partial_{t} \vartheta_{N}=-\vartheta_{N}+\Delta \vartheta_{N}-\int_{0}^{\infty} \nu(\sigma) \eta_{N}(\sigma) d \sigma+\int_{0}^{\infty} \mu(\sigma) \Delta \eta_{N}(\sigma) d \sigma-\lambda^{\prime}(\chi) \chi_{t}$

(7.15) $\partial_{t} \chi_{N}=-\chi_{N}+\Delta \chi_{N}-\chi^{3}+\tilde{\gamma}(\chi)+\lambda^{\prime}(\chi) \vartheta$

(7.14) $\partial_{t} \eta_{N}+\partial_{s} \eta_{N}=\vartheta_{N}$

(7.16) $z_{N}(\tau)=0$. 
Fix $\tau \in \mathbb{R}$, and let $t \geq \tau+1$. Throughout the proof, $c$ will depend only on $R_{0}$ and $\|g\|_{\mathcal{T}_{1}}$. To get estimate (7.13) we will proceed formally. Of course, the argument can be justified using the Faedo-Galerkin scheme introduced in Section 4 (see 4.2). Take the inner product in $H$ of (7.14) and $\vartheta_{N}$ and $-\Delta \vartheta_{N}$, respectively. Adding the results,

$$
\begin{aligned}
\frac{1}{2} \frac{d}{d t} & \left(\left\|\vartheta_{N}\right\|_{H}^{2}+\left\|\nabla \vartheta_{N}\right\|_{H^{3}}^{2}\right)+\left\|\vartheta_{N}\right\|_{H}^{2}+2\left\|\nabla \vartheta_{N}\right\|_{H^{3}}^{2}+\left\|\Delta \vartheta_{N}\right\|_{H}^{2} \\
= & -\left\langle\eta_{N}, \vartheta_{N}\right\rangle_{\mathcal{L}}+\int_{0}^{\infty} \mu(\sigma)\left\langle\eta_{N}(\sigma), \vartheta_{N}\right\rangle_{H} d \sigma-\left\langle\lambda^{\prime}(\chi) \chi_{t}, \vartheta_{N}\right\rangle_{H} \\
& +\left\langle\lambda^{\prime}(\chi) \chi_{t}, \Delta \vartheta_{N}\right\rangle_{H}
\end{aligned}
$$

Lemma 6.6 and (4.11) entail

$$
\left\|\lambda^{\prime}(\chi) \chi_{t}\right\|_{H} \leq\left\|\lambda^{\prime}(\chi)\right\|_{L^{\infty}(\Omega)}\left\|\chi_{t}\right\|_{H} \leq c \quad \text { for all } t \geq \tau+1
$$

In light of Lemma 6.2, Lemma 7.4, and (7.12), it is also apparent that

$$
\left\|z_{N}(t)\right\|_{\mathcal{H}} \leq c \quad \text { for all } t \geq \tau
$$

Thus, from Young inequality,

$$
\begin{gathered}
\int_{0}^{\infty} \mu(\sigma)\left\langle\eta_{N}(\sigma), \vartheta_{N}\right\rangle_{H} d \sigma-\left\langle\lambda^{\prime}(\chi) \chi_{t}, \vartheta_{N}\right\rangle_{H} \\
+\left\langle\lambda^{\prime}(\chi) \chi_{t}, \Delta \vartheta_{N}\right\rangle_{H} \leq c+\left\|\Delta \vartheta_{N}\right\|_{H}^{2}
\end{gathered}
$$

Then take the inner product in $\mathcal{L}$ of (7.16) and $\eta_{N}$ and integrate by parts using (K4), to get

$$
\frac{1}{2} \frac{d}{d t}\left\|\eta_{N}\right\|_{\mathcal{L}}^{2}+\frac{\delta}{2}\left\|\eta_{N}\right\|_{\mathcal{L}}^{2} \leq\left\langle\eta_{N}, \vartheta_{N}\right\rangle_{\mathcal{L}}
$$

Summation of (7.18) and (7.21), with the aid of (7.20), bears

$$
\frac{1}{2} \frac{d}{d t}\left(\left\|\vartheta_{N}\right\|_{V}^{2}+\left\|\eta_{N}\right\|_{\mathcal{L}}^{2}\right)+\left\|\vartheta_{N}\right\|_{V}^{2}+\frac{\delta}{2}\left\|\eta_{N}\right\|_{\mathcal{L}}^{2} \leq c
$$

and we get at once the thesis for the first and the third component of $z_{N}$. Finally, denote

$$
\Phi^{2}(t)=\left\|\chi_{N}\right\|_{H}^{2}+\frac{3}{2}\left\|\nabla \chi_{N}\right\|_{H^{3}}^{2}+\frac{1}{2}\left\|\Delta \chi_{N}\right\|_{H}^{2}
$$


and take the inner product in $H$ of (7.15) with $\chi_{N}, \partial_{t} \chi_{N},-\Delta \chi_{N}$, and $-\partial_{t} \Delta \chi_{N}$, respectively. Adding the resulting equations we get

$$
\begin{array}{r}
\frac{d}{d t} \Phi^{2}+\Phi^{2}+\frac{1}{2}\left(\left\|\nabla \chi_{N}\right\|_{H^{3}}^{2}+\left\|\Delta \chi_{N}\right\|_{H}^{2}\right) \\
+\left\|\partial_{t} \chi_{N}\right\|_{H}^{2}+\left\|\partial_{t} \nabla \chi_{N}\right\|_{H^{3}}^{2}=\sum_{j=1}^{4} I_{j}
\end{array}
$$

with

$$
\begin{aligned}
I_{1}= & -\left\langle\chi^{3}, \chi_{N}\right\rangle_{H}+\left\langle\tilde{\gamma}(\chi), \chi_{N}\right\rangle_{H}+\left\langle\lambda^{\prime}(\chi) \vartheta, \chi_{N}\right\rangle_{H} \\
I_{2}=- & \left\langle\chi^{3}, \partial_{t} \chi_{N}\right\rangle_{H}+\left\langle\tilde{\gamma}(\chi), \partial_{t} \chi_{N}\right\rangle_{H}+\left\langle\lambda^{\prime}(\chi) \vartheta, \partial_{t} \chi_{N}\right\rangle_{H} \\
I_{3}= & \left\langle\chi^{3}, \Delta \chi_{N}\right\rangle_{H}-\left\langle\tilde{\gamma}(\chi), \Delta \chi_{N}\right\rangle_{H}-\left\langle\lambda^{\prime}(\chi) \vartheta, \Delta \chi_{N}\right\rangle_{H} \\
I_{4}=- & 3\left\langle\chi^{2} \nabla \chi, \partial_{t} \nabla \chi_{N}\right\rangle_{H^{3}}+\left\langle\tilde{\gamma}^{\prime}(\chi) \nabla \chi, \partial_{t} \nabla \chi_{N}\right\rangle_{H^{3}} \\
& \quad+\left\langle\lambda^{\prime}(\chi) \nabla \vartheta, \partial_{t} \nabla \chi_{N}\right\rangle_{H^{3}}+\left\langle\lambda^{\prime \prime}(\chi) \nabla \chi \vartheta, \partial_{t} \nabla \chi_{N}\right\rangle_{H^{3}} .
\end{aligned}
$$

Using (7.19) and Lemma 6.6 (which, in particular, ensures that $\left\|\lambda^{\prime}(\chi) \vartheta\right\|_{H} \leq c$ ), one easily sees that

$$
I_{1}+I_{2}+I_{3} \leq \frac{1}{2}\left\|\nabla \chi_{N}\right\|_{H^{3}}^{2}+\left\|\partial_{t} \chi_{N}\right\|_{H}^{2}+\frac{1}{2}\left\|\Delta \chi_{N}\right\|_{H}^{2}+c .
$$

Concerning $I_{4}$, one can repeat the calculations of Lemma 6.6, recalling that now (since Lemma 6.6 holds) $\|\chi\|_{W} \leq c$. Therefore

$$
I_{4} \leq c+c\|\vartheta\|_{V}^{2}+\left\|\partial_{t} \nabla \chi_{N}\right\|_{H^{3}}^{2} .
$$

Hence

$$
\sum_{j=1}^{4} I_{j} \leq \frac{1}{2}\left\|\nabla \chi_{N}\right\|_{H^{3}}^{2}+\left\|\partial_{t} \chi_{N}\right\|_{H}^{2}+\frac{1}{2}\left\|\Delta \chi_{N}\right\|_{H}^{2}+\left\|\partial_{t} \nabla \chi_{N}\right\|_{H^{3}}^{2}+c+c\|\vartheta\|_{V}^{2}
$$

and (7.22) turns into

$$
\frac{d}{d t} \Phi^{2}+\frac{1}{2} \Phi^{2} \leq c\left(1+\|\vartheta\|_{V}^{2}\right) .
$$

But, from Lemma 6.2 and Lemma 6.3, $\sup _{t \geq \tau+1} \int_{t}^{t+1}\|\vartheta(y)\|_{V}^{2} d y<\infty$, and the conclusion of the proof follows from Lemma 2.5 (applied for $t \geq \tau+1$ ). Indeed, $\Phi(\tau)=0$, in virtue of $(7.17)$, and $\left\|\chi_{N}\right\|_{W}^{2} \leq 2 \Phi^{2}$. 
Our aim is to show that $z_{N}$ runs in a compact subset of $\mathcal{H}$. Unfortunately, the embedding $\mathcal{V} \hookrightarrow \mathcal{H}$ is not compact, since the embedding $\mathcal{L} \hookrightarrow \mathcal{M}$ is not. Let us dwell for a moment on the third component $\eta_{N}$ of $z_{N}$.

Lemma 7.7. The set

$$
\mathcal{E}=\bigcup_{f \in \mathrm{H}(g)} \bigcup_{z_{0} \in \mathcal{B}_{0}} \bigcup_{\tau \in \mathbb{R}} \bigcup_{t \geq \tau} \eta_{N}^{t}
$$

is relatively compact in $\mathcal{M}$.

Proof. It is clear from Lemma 7.6 that $\mathcal{E}$ is bounded in $L_{\nu}^{2}\left(\mathbb{R}^{+}, V\right) \cap$ $L_{\mu}^{2}\left(\mathbb{R}^{+}, W\right)$. Observe that $\eta_{N}$ can be computed explicitly from (7.6) as follows (cf. $(4.55))$

$$
\eta_{N}^{t}(s)= \begin{cases}\int_{0}^{s} \vartheta_{N}(t-y) d y & 0<s \leq t-\tau, \\ \int_{0}^{t-\tau} \vartheta_{N}(t-y) d y & s>t-\tau .\end{cases}
$$

Differentiating (7.23) with respect to $s$ yields

$$
\partial_{s} \eta_{N}^{t}(s)= \begin{cases}\vartheta_{N}(t-s) & 0<s \leq t-\tau, \\ 0 & s>t-\tau .\end{cases}
$$

Therefore (7.19) and (7.24) give at once

$$
\int_{0}^{\infty} \mu(s)\left\|\partial_{s} \eta_{N}^{t}(s)\right\|_{H}^{2} d s=\int_{0}^{t-\tau} \mu(s)\left\|\vartheta_{N}(t-s)\right\|_{H}^{2} d s \leq c
$$

and, analogously

$$
\int_{0}^{\infty} \nu(s)\left\|\partial_{s} \eta_{N}^{t}(s)\right\|_{H}^{2} d s \leq c .
$$

We conclude that $\mathcal{E}$ is bounded in

$$
L_{\nu}^{2}\left(\mathbb{R}^{+}, V\right) \cap H_{\nu}^{1}\left(\mathbb{R}^{+}, H\right) \cap L_{\mu}^{2}\left(\mathbb{R}^{+}, W\right) \cap H_{\mu}^{1}\left(\mathbb{R}^{+}, H\right) .
$$


Finally, from (7.23),

$(7.25)\left\|\eta_{N}^{t}(s)\right\|_{V} \leq \begin{cases}\int_{t-s}^{t}\left\|\vartheta_{N}(y)\right\|_{V} d y, & \tau+1 \leq t-s<t, \\ \int_{t-s}^{\tau+1}\left\|\vartheta_{N}(y)\right\|_{V} d y+\int_{\tau+1}^{t}\left\|\vartheta_{N}(y)\right\|_{V} d y, & \tau \leq t-s<\tau+1, \\ \int_{\tau}^{\tau+1}\left\|\vartheta_{N}(y)\right\|_{V} d y+\int_{\tau+1}^{t}\left\|\vartheta_{N}(y)\right\|_{V} d y, & t-s<\tau .\end{cases}$

Notice that, integration of (7.8) over $[t, t+1]$ and Lemma 6.2 entail

$$
\sup _{\tau \in \mathbb{R}} \sup _{t \geq \tau} \int_{t}^{t+1}\left\|\vartheta_{N}(y)\right\|_{V} d y \leq c .
$$

Thus, from (7.13) and (7.25)-(7.26),

$$
\left\|\eta_{N}(s)\right\|_{V}^{2} \leq(c+K s)^{2} \in L_{\nu}^{1}\left(\mathbb{R}^{+}\right) \cap L_{\mu}^{1}\left(\mathbb{R}^{+}\right)
$$

in force of the exponential decays of $\nu$ and $\mu$. Applying Lemma 2.2, we get the thesis (notice that $c$ depends only on $R_{0}$ and $\|g\|_{\mathcal{T}_{1}}$ ).

Denote now $\mathcal{K}_{N}=\mathcal{B}_{K} \times \overline{\mathcal{E}}$, where $\mathcal{B}_{K}$ is the closed ball of $V \times W$ of radius $K$, and $\overline{\mathcal{E}}$ is the norm-closure of $\mathcal{E}$ in $\mathcal{M}$. Then, from the compact embedding $V \times W \hookrightarrow H \times V$, Lemma 7.6, and Lemma 7.7, we conclude that $\mathcal{K}_{N}$ is compact in $\mathcal{H}$. Finally, define

$$
\mathcal{K}=\mathcal{K}_{E}+\mathcal{K}_{N} \subset \mathcal{H}
$$

Since $\mathcal{K}_{E}$ (from Lemma 7.5) and $\mathcal{K}_{N}$ are compact, $\mathcal{K}$ is compact. Moreover, by construction,

$$
\bigcup_{f \in \mathrm{H}(g)} \bigcup_{z_{0} \in \mathcal{B}_{0}} \bigcup_{\tau \in \mathbb{R}} \bigcup_{t \geq \tau+1}\left(z_{E}(t)+z_{N}(t)\right) \subset \mathcal{K} .
$$

We are now able to state and prove the main result of the paper.

Theorem 7.8. Let (K1)-(K4), (H1)-(H2), (H4)-(H6) hold and let $g$ be a translation compact function in $L_{\mathrm{loc}}^{1}(\mathbb{R}, H)$. Then the family of processes $\left\{U_{f}(t, \tau), f \in \mathrm{H}(g)\right\}$ associated with $\mathbf{P}$ possesses a compact uniform attractor $\mathcal{A}$ which has the explicit form given by (7.2). 
Proof. The proof of the continuity of $U_{\bullet}(t, \tau)$ as a map $\mathcal{H} \times \mathrm{H}(g) \rightarrow \mathcal{H}$ follows from Theorem 3.2 and Theorem 3.4. Thus, in view of Theorem 7.3, we are left to show that the set $\mathcal{K}$ given by (7.27) is uniformly attracting for the family $\left\{U_{f}(t, \tau), f \in \mathrm{H}(g)\right\}$. Let $\mathcal{B} \subset \mathcal{H}$ be a bounded set, and let $t_{0}=t_{0}(\mathcal{B})$ be as in Definition 6.1. There is no harm to suppose $t_{0} \geq 1$. For any $t_{1}>0$, and every $\tau \in \mathbb{R}$, we have

$$
\begin{aligned}
U_{f}\left(t_{1}+t_{0}+\tau, \tau\right) \mathcal{B} & =U_{f}\left(t_{1}+t_{0}+\tau, t_{0}+\tau\right) U_{f}\left(t_{0}+\tau, \tau\right) \mathcal{B} \\
& \subset U_{f}\left(t_{1}+t_{0}+\tau, t_{0}+\tau\right) \mathcal{B}_{0}
\end{aligned}
$$

By Lemma 7.4 and (7.28),

$$
\sup _{f \in \mathrm{H}(g)} \operatorname{dist}\left(U_{f}(t, \tau) \mathcal{B}, \mathcal{K}\right) \leq M R_{0} e^{\varepsilon_{0}\left(t_{0}+\tau\right)} e^{-\varepsilon_{0} t}
$$

for every $t \geq t_{0}+\tau$, and (7.1) follows at once.

Proposition 7.9. The uniform attractor $\mathcal{A}$ for the family $\left\{U_{f}(t, \tau), f \in\right.$ $\mathrm{H}(g)$ \} given by Theorem 7.8 is connected.

Proof. The set $\mathcal{A} \times \mathrm{H}(g)$ turns out to be the global attractor of the semigroup $V(t)$ on the complete metric space $\mathcal{H} \times \mathrm{H}(g)$ defined by

$$
V(t)(z, f)=\left(U_{f}(t, 0) z, f^{t}\right)
$$

(see [CV1] for more details). In the course of the investigation, we showed that $\mathcal{B}_{0} \times \mathrm{H}(g)$ is a bounded absorbing set for the semigroup $V(t)$ defined above. In particular, $\mathcal{B}_{0}$ can be chosen to be a ball. Moreover, it is immediate to see that $\left\{g^{r}\right\}_{r \in \mathbb{R}}$ is path connected, and therefore its closure $\mathrm{H}(g)$ is connected; we conclude that $\mathcal{B}_{0} \times \mathrm{H}(g)$ is connected. Hence (see, e.g., [Har], Proposition 5.2.7) the attractor $\mathcal{A} \times \mathrm{H}(g)$ of $V(t)$ is connected too, and so is its projection on $\mathcal{H}$, that is, $\mathcal{A}$.

\section{Finite Dimension of the Attractor}

Here, we prove that the uniform attractor found in the previous section has finite Hausdorff and fractal dimensions, provided that $\lambda$ is linear.

We recall that the Hausdorff dimension of a subset $\mathcal{X} \subset \mathcal{H}$ is defined by

$$
\operatorname{dim}_{\mathrm{H}} \mathcal{X}=\sup \left\{d>0: \sup _{\varepsilon>0} \inf _{C_{\varepsilon}} \sum_{i \in J} r_{i}^{d}<\infty\right\}
$$

where $C_{\varepsilon}=\left\{\mathcal{B}_{i}\left(r_{i}\right)\right\}_{i \in J}$ is a covering of $\mathcal{X}$ of balls of radii $r_{i} \leq \varepsilon$. The fractal dimension of $\mathcal{X}$ is defined by

$$
\operatorname{dim}_{\mathrm{F}} \mathcal{X}=\sup \left\{d>0: \limsup _{\varepsilon>0} \varepsilon^{d} n_{\mathcal{X}}(\varepsilon)<\infty\right\},
$$


where $n_{\mathcal{X}}(\varepsilon)$ is the minimum number of balls of radius $\varepsilon$ which is necessary to cover $\mathcal{X}$. It follows directly from the definition that

$$
\operatorname{dim}_{\mathrm{H}} \mathcal{X} \leq \operatorname{dim}_{\mathrm{F}} \mathcal{X}
$$

Let us replace conditions (H1)-(H3) with

$$
\begin{aligned}
& \gamma \in C^{2}(\mathbb{R}) \quad \text { and } \quad \gamma^{\prime} \in L^{\infty}(\mathbb{R}) \\
& \lambda(r)=\lambda_{0} r, \quad \lambda_{0} \in \mathbb{R} \\
& f \in H
\end{aligned}
$$

When (H9) holds, the dynamical system is autonomous and $U_{f}(t, 0)$ is a strongly continuous semigroup on $\mathcal{H}$. Setting $\omega=\vartheta+\lambda_{0} \chi$ and introducing the triplets

$$
\zeta_{0}=\left(\omega_{0}, \chi_{0}, \eta_{0}\right) \quad \text { and } \quad \zeta(t)=\left(\omega(t), \chi(t), \eta^{t}\right)
$$

we can rewrite problem $\mathbf{P}$ as

$$
\begin{aligned}
\zeta_{t}= & L_{1} z+N_{1}(\zeta), \quad \zeta(\tau)=\zeta_{0} \\
& + \text { boundary conditions }
\end{aligned}
$$

where the linear operator $L_{1}$ has the (formal) expression

$$
L_{1}=\left(\begin{array}{ccc}
-\mathbb{I}+\Delta & \lambda_{0} \mathbb{I}-\lambda_{0} \Delta & -\int_{0}^{\infty} \nu(\sigma) \mathbb{I} \cdot d \sigma+\int_{0}^{\infty} \mu(\sigma) \Delta \cdot d \sigma \\
\lambda_{0} \mathbb{I} & -\lambda_{0}^{2} \mathbb{I}+\Delta & 0 \\
\mathbb{I} & -\lambda_{0} \mathbb{I} & -\partial_{s}
\end{array}\right)
$$

and

$$
N_{1}(\zeta)=(f, \beta(\chi), 0)
$$

having set $\beta(r)=-r^{3}+\gamma(r)$. Note that, from $(\mathrm{H} 7), \beta \in C^{2}(\mathbb{R})$. Clearly, the boundary conditions might be read defining properly the domain of the operator $L_{1}$.

System (8.1) generates a strongly continuous semigroup $U(t)$ on $\mathcal{H}$, which is related to $U_{f}(t, 0)$ as follows

$$
U(t) \zeta_{0}=U_{f}(t, 0) z_{0} \quad \text { whenever } \quad \omega_{0}=\vartheta_{0}+\lambda_{0} \chi_{0} .
$$

Therefore, all the previous results hold for $U(t)$ as well. In particular, the set

$$
\mathcal{A}_{1}=\left\{\left(\vartheta_{0}+\lambda_{0} \chi_{0}, \chi_{0}, \eta_{0}\right) \text { such that }\left(\vartheta_{0}, \chi_{0}, \eta_{0}\right) \in \mathcal{A} t\right\}
$$


is the global attractor of $U(t)$ on $\mathcal{H}$.

Let now $z_{0}=\left(\vartheta_{0}, \chi_{0}, \eta_{0}\right) \in \mathcal{H}$ be fixed, and let $\zeta_{0}=\left(\omega_{0}+\lambda_{0} \chi_{0}, \chi_{0}, \eta_{0}\right)$. Select an arbitrary $r>0$, and let $z=(\vartheta, \chi, \eta) \in \mathcal{H}$ be such that $\left\|z-z_{0}\right\|_{\mathcal{H}} \leq r$. Then, setting $\zeta=\left(\omega+\lambda_{0} \chi, \chi, \eta\right)$, we easily get that $\left\|\zeta-\zeta_{0}\right\|_{\mathcal{H}} \leq c r$ for some $c>0$. Thus, $n_{\mathcal{A}}(c r) \leq n_{\mathcal{A}_{1}}(r)$, which entails $\operatorname{dim}_{\mathrm{F}} \mathcal{A} \leq \operatorname{dim}_{\mathrm{F}} \mathcal{A}_{1}$. Hence, in order to show that $\operatorname{dim}_{\mathrm{F}} \mathcal{A}<\infty$, we will proceed showing that $\operatorname{dim}_{\mathrm{F}} \mathcal{A}_{1}<\infty$.

Before going any further, we recall some definitions and a basic result due to Constantin, Foias and Temam [CFT]. Let $B(\mathcal{H})$ denote the space of bounded linear operators on $\mathcal{H}$.

Definition 8.1. $A$ (nonlinear) continuous map $U$ from a subset $\mathcal{X} \subset \mathcal{H}$ into $\mathcal{H}$ is said to be uniformly quasidifferentiable on $\mathcal{X}$ if for any $\zeta_{0} \in \mathcal{X}$ there exists $U^{\prime}\left(\zeta_{0}\right) \in B(\mathcal{H})$ (the quasidifferential of $U$ at $\zeta_{0}$ with respect to $\mathcal{H}$ ) such that

$$
\left\|U \zeta_{0}-U \zeta-U^{\prime}\left(\zeta_{0}\right)\left(\zeta_{0}-\zeta\right)\right\|_{\mathcal{H}} \leq \sigma\left(\left\|\zeta_{0}-\zeta\right\|_{\mathcal{H}}\right)\left\|\zeta_{0}-\zeta\right\|_{\mathcal{H}} \quad \text { for all } \zeta \in \mathcal{X}
$$

where $\sigma: \mathbb{R} \rightarrow \mathbb{R}^{+}$is independent of $\zeta_{0}$, and $\sigma(y) \rightarrow 0$ as $y \rightarrow 0^{+}$. The operator $U^{\prime}\left(\zeta_{0}\right)$ might not be unique.

Definition 8.2. Let $M$ be a linear operator on $\mathcal{H}$. For any positive integer $m$, the $m$-dimensional trace of $M$ is defined as

$$
\operatorname{Tr}_{m} M=\sup _{Q} \sum_{j=1}^{m}\left\langle M \zeta_{j}, \zeta_{j}\right\rangle_{\mathcal{H}}
$$

where the supremum ranges over all possible orthogonal projections $Q$ in $\mathcal{H}$ on the $m$-dimensional space $Q \mathcal{H}$ belonging to the domain of $M$, and $\left\{\zeta_{1}, \ldots, \zeta_{m}\right\}$ is an orthonormal basis of $Q \mathcal{H}$.

Theorem 8.3. Let $\mathcal{X} \subset \mathcal{H}$ be a compact fully invariant set for $U(t)$, i.e., $U(t) \mathcal{X}=\mathcal{X}$ for all $t \geq 0$. Assume also that $U(t)$ is uniformly quasidifferentiable on $\mathcal{X}$ for all $t \geq 0$, and

$$
\sup _{\zeta_{0} \in \mathcal{X}}\left\|U^{\prime}\left(t, \zeta_{0}\right)\right\|_{B(\mathcal{H})}<\infty \quad \text { for all } t \geq 0
$$

where $U^{\prime}\left(t, \zeta_{0}\right)$ is the quasidifferential of $U(t)$ at $\zeta_{0}$. It is also assumed that $U^{\prime}\left(t, \zeta_{0}\right)$ is generated by the equation in variation

$$
Z_{t}=M(\zeta) Z, \quad Z(0)=Z_{0}
$$

that is, $U^{\prime}\left(t, \zeta_{0}\right) Z_{0}=Z(t)$, with $\zeta(t)=U(t) \zeta_{0}$, where $M(\zeta)$ is a linear operator 
on $\mathcal{H}$, for every fixed $\zeta \in \mathcal{X}$. Introducing the number $q_{m}$ by the formula

$$
q_{m}=\liminf _{T \rightarrow \infty} \sup _{\zeta_{0} \in \mathcal{X}}\left\{\frac{1}{T} \int_{0}^{T} \operatorname{Tr}_{m} M\left(U(t) \zeta_{0}\right) d t\right\}
$$

if there exists $m \geq 2$ such that $q_{m}<0$, then

$$
\operatorname{dim}_{\mathrm{H}} \mathcal{X} \leq m \quad \text { and } \quad \operatorname{dim}_{\mathrm{F}} \mathcal{X} \leq m \max _{1 \leq j \leq m-1}\left(1+\frac{\max \left\{q_{j}, 0\right\}}{\left|q_{m}\right|}\right)
$$

We now apply this general result to system (8.1).

Proposition 8.4. Let (K1)-(K4) and (H4)-(H9) hold. Then the semigroup $U(t)$ acting on $\mathcal{H}$ is uniformly quasidifferentiable on $\mathcal{A}_{1}$ for every $t \geq 0$, and the quasidifferential $U^{\prime}\left(t, \zeta_{0}\right)$ at the point $\zeta_{0}=\left(\omega_{0}, \chi_{0}, \eta_{0}\right) \in \mathcal{A}_{1}$ satisfies the equation in variation

$$
Z_{t}=L_{1} Z+N_{1}^{\prime}(\zeta) Z, \quad Z(0)=Z_{0}
$$

where

$$
\begin{gathered}
U^{\prime}\left(t, \zeta_{0}\right) Z_{0}=Z(t)=\left(\Theta(t), X(t), Y^{t}\right), \\
\zeta(t)=\left(\omega(t), \chi(t), \eta^{t}\right)=U(t) \zeta_{0},
\end{gathered}
$$

and

$$
N_{1}^{\prime}(\zeta) Z=\left(0, \beta^{\prime}(\chi) X, 0\right)
$$

being $N_{1}^{\prime}$ the Fréchet differential of $N_{1}$. Furthermore

$$
\sup _{\zeta_{0} \in \mathcal{A}_{1}}\left\|U^{\prime}\left(t, \zeta_{0}\right)\right\|_{B(\mathcal{H})}<\infty \quad \text { for all } t \geq 0
$$

Proof. Arguing as in Theorem 3.2 and Theorem 3.4, it is possible to show that $(8.2)$ has a unique solution $Z \in C^{0}\left(\mathbb{R}^{+}, \mathcal{H}\right)$. Notice that $(8.2)$ is a linear (non-autonomous) system.

Let now $\zeta$ and $\zeta^{*}$ be solutions to problem (8.1) with initial data $\zeta_{0}$ and $\zeta_{0}^{*}$, respectively, with $\zeta_{0}, \zeta_{0}^{*} \in \mathcal{A}_{1}$. The difference $\tilde{\zeta}=(\tilde{\omega}, \tilde{\chi}, \tilde{\eta})=\zeta^{*}-\zeta$ satisfies the problem

$$
\begin{aligned}
\tilde{\zeta}_{t} & =L_{1} \tilde{\zeta}+N_{1}\left(\zeta^{*}\right)-N_{1}(\zeta) \\
\tilde{\zeta}(0) & =\tilde{\zeta}_{0}=\zeta_{0}^{*}-\zeta_{0} .
\end{aligned}
$$


Pick $T \in \mathbb{R}^{+}$. In the remaining of the proof, the generic constant $c$ will depend on $T$. Recalling estimate (3.16), we have

$$
\|\tilde{\zeta}(t)\|_{\mathcal{H}}^{2} \leq c\left\|\tilde{\zeta}_{0}\right\|_{\mathcal{H}}^{2} \quad \text { for all } t \in[0, T]
$$

Finally, let $Z$ be the solution to (8.2) with initial data $Z(0)=\tilde{\zeta}_{0}$. Our aim is to show that $Z(t)=U^{\prime}\left(t, \zeta_{0}\right) \tilde{\zeta}_{0}$. Set

$$
\xi=\zeta^{*}-\zeta-Z=\tilde{\zeta}-Z
$$

Clearly, $\xi$ satisfies

$$
\xi_{t}=L_{1} \xi+N_{1}^{\prime}(\zeta) \xi-h\left(\chi^{*}, \chi\right), \quad \xi(0)=0
$$

where

$$
h\left(\chi^{*}, \chi\right)=\left(0, \beta(\chi)-\beta\left(\chi^{*}\right)+\beta^{\prime}(\chi) \tilde{\chi}, 0\right) .
$$

As a consequence of Lemma 6.6, the projection of $\mathcal{A}_{1}$ on $V$ is bounded in $L^{\infty}(\Omega)$. Indeed, due to the embedding $W \hookrightarrow L^{\infty}(\Omega)$, we get at once that the projection on $V$ of $U(1) \mathcal{A}_{1}$ is bounded, but for the invariance property of the attractor, $\mathcal{A}_{1}=U(1) \mathcal{A}_{1}$. Exploiting the argument used in Theorem 6.4 of [PZ] (based on the uniform continuity of $\beta^{\prime}$ on closed intervals), and (8.3), it is not hard to see that

$$
\left\|\beta(\chi)-\beta\left(\chi^{*}\right)+\beta^{\prime}(\chi) \tilde{\chi}\right\|_{H}^{2} \leq\left\|\tilde{\zeta}_{0}\right\|_{\mathcal{H}}^{2} \sigma\left(\left\|\tilde{\zeta}_{0}\right\|_{\mathcal{H}}\right)
$$

where $\sigma(y) \downarrow 0$ as $y \downarrow 0$. Multiply now (8.4) by $\xi$, to get

$$
\frac{d}{d t}\|\xi\|_{\mathcal{H}}^{2}=2\left\langle L_{1} \xi, \xi\right\rangle_{\mathcal{H}}+2\left\langle N_{1}^{\prime}(\zeta) \xi, \xi\right\rangle_{\mathcal{H}}+2\left\langle h\left(u^{*}, u\right), \xi\right\rangle_{\mathcal{H}}
$$

Denoting $\xi=\left(\xi_{1}, \xi_{2}, \xi_{3}\right)$, applying repeatedly Young inequality one can show show that

$$
2\left\langle L_{1} \xi, \xi\right\rangle_{\mathcal{H}} \leq-\delta_{0}\left(\left\|\xi_{1}\right\|_{V}^{2}+\left\|\xi_{2}\right\|_{W}^{2}+\left\|\xi_{3}\right\|_{\mathcal{M}}^{2}\right)+c\left(\left\|\xi_{1}\right\|_{H}^{2}+\left\|\xi_{2}\right\|_{V}^{2}\right)
$$

for any fixed $\delta_{0}<\min \{2, \delta\}$. Moreover, since $\chi$ is bounded in $L^{\infty}(\Omega)$,

$$
\begin{aligned}
2\left\langle N_{1}^{\prime}(\zeta) \xi, \xi\right\rangle_{\mathcal{H}}= & 2\left\langle\beta^{\prime}(\chi) \xi_{2}, \xi_{2}\right\rangle_{H}+2\left\langle\beta^{\prime}(\chi) \nabla \xi_{2}, \nabla \xi_{2}\right\rangle_{H} \\
& +2\left\langle\beta^{\prime \prime}(\chi) \nabla \chi \xi_{2}, \nabla \xi_{2}\right\rangle_{H} \\
\leq & c\left\|\xi_{2}\right\|_{V}^{2}+c\|\nabla \chi\|_{L^{3}(\Omega)}\left\|\xi_{2}\right\|_{L^{6}(\Omega)}\left\|\nabla \xi_{2}\right\|_{H} \leq c\|\xi\|_{\mathcal{H}^{\prime}}^{2}
\end{aligned}
$$


For further use, notice that the constant $c$ appearing in (8.7) and (8.8) is independent of $T$ (though it depends on $\delta_{0}$ ). Finally, from (8.5),

$$
\begin{aligned}
2\left\langle h\left(\chi^{*}, \chi\right), \xi\right\rangle_{\mathcal{H}} & =2\left\langle\beta(\chi)-\beta\left(\chi^{*}\right)+\beta^{\prime}(\chi) \tilde{\chi}, \xi_{2}\right\rangle_{V} \\
& \leq 2\left\|\beta(\chi)-\beta\left(\chi^{*}\right)+\beta^{\prime}(\chi) \tilde{\chi}\right\|_{H}\left\|\xi_{2}\right\|_{W} \\
& \leq c\left\|\beta(\chi)-\beta\left(\chi^{*}\right)+\beta^{\prime}(\chi) \tilde{\chi}\right\|_{H}^{2}+\delta_{0}\left\|\xi_{2}\right\|_{W}^{2} \\
& \leq c\left\|\tilde{\zeta}_{0}\right\|_{\mathcal{H}}^{2} \sigma\left(\left\|\tilde{\zeta}_{0}\right\|_{\mathcal{H}}\right)+\delta_{0}\left\|\xi_{2}\right\|_{W}^{2} .
\end{aligned}
$$

Collecting (8.7)-(8.9), inequality (8.6) turns into

$$
\frac{d}{d t}\|\xi\|_{\mathcal{H}}^{2} \leq c\|\xi\|_{\mathcal{H}}^{2}+c\left\|\tilde{\zeta}_{0}\right\|_{\mathcal{H}}^{2} \sigma\left(\left\|\tilde{\zeta}_{0}\right\|_{\mathcal{H}}\right)
$$

and Gronwall Lemma yields

$$
\frac{\|\xi(T)\|_{\mathcal{H}}^{2}}{\left\|\tilde{\zeta}_{0}\right\|_{\mathcal{H}}^{2}} \leq c \sigma\left(\left\|\tilde{\zeta}_{0}\right\|_{\mathcal{H}}\right)
$$

We conclude that, for every $T \in \mathbb{R}^{+}$,

$$
\lim _{\left\|\tilde{\zeta}_{0}\right\|_{\mathcal{H}} \rightarrow 0} \frac{\|\xi(T)\|_{\mathcal{H}}}{\left\|\tilde{\zeta}_{0}\right\|_{\mathcal{H}}}=0
$$

which entails the required differentiability. Finally, taking the inner product of (8.2) and $Z$, and performing similar calculations, we obtain the estimate

$$
\|Z(T)\|_{\mathcal{H}}^{2} \leq c\left\|Z_{0}\right\|_{\mathcal{H}}^{2}
$$

which yields the last assertion of the proposition.

We now report a classical result, which will be exploited in the next theorem (for the proof see $[\mathrm{CH}]$ and Lemma VI.2.1 in $[\mathrm{Tem}]$ ).

Lemma 8.5. There exists a positive constant $\ell$ such that, for any given $m$ vectors $\left\{v_{1}, \ldots, v_{m}\right\}$ in $V$ and $\left\{w_{1}, \ldots, w_{m}\right\}$ in $W$ which are orthonormal in $H$ and in $V$, respectively, it follows that

$$
\sum_{j=1}^{m}\left\|v_{j}\right\|_{V}^{2} \geq \ell m^{5 / 3} \quad \text { and } \quad \sum_{j=1}^{m}\left\|w_{j}\right\|_{W}^{2} \geq \ell m^{5 / 3}
$$


Theorem 8.6. Let (K1)-(K4) and (H4)-(H9) hold. Then the attractor $\mathcal{A}$ of the semigroup $U_{f}(t, 0)$ acting on $\mathcal{H}$ has finite fractal (and Hausdorff) dimension.

Proof. As anticipated, we will show that the attractor $\mathcal{A}_{1}$ of the semigroup $U(t)$ acting on $\mathcal{H}$ has finite fractal dimension. Let $\zeta_{0} \in \mathcal{A}_{1}$ (so that $\zeta(t) \in \mathcal{A}_{1}$ for every $t \geq 0)$. Given any unitary vector $Z=(\Theta, X, Y)$ belonging to the domain of $L_{1}+N_{1}^{\prime}(\zeta)$, using (8.7)-(8.8), we have

$$
\left\langle\left(L_{1}+N_{1}^{\prime}(\zeta)\right) Z, Z\right\rangle_{\mathcal{H}} \leq-\delta_{0}\left(\|\Theta\|_{V}^{2}+\|X\|_{W}^{2}+\|Y\|_{\mathcal{M}}^{2}\right)+c\left(\|\Theta\|_{H}^{2}+\|X\|_{V}^{2}\right)
$$

Therefore we conclude that $L_{1}+N_{1}^{\prime}(\zeta) \leq M$, where $M$ is the diagonal operator acting on $H \oplus V \oplus \mathcal{M}$ defined by

$$
M=\left(\begin{array}{ccc}
c \mathbb{I}-\delta_{0}(\mathbb{I}-\Delta) & 0 & 0 \\
0 & c \mathbb{I}-\delta_{0}(\mathbb{I}-\Delta) & 0 \\
0 & 0 & -\delta_{0} \mathbb{I}
\end{array}\right)
$$

Directly from the definition of $\operatorname{Tr}_{m}$, it is apparent that $\operatorname{Tr}_{m}\left(L_{1}+N_{1}^{\prime}(\zeta)\right) \leq$ $\operatorname{Tr}_{m}(M)$. Finally, since $M$ is diagonal,

$$
\operatorname{Tr}_{m}(M)=\sup _{Q} \sum_{j=1}^{m}\left\langle M Z_{j}, Z_{j}\right\rangle_{\mathcal{H}}
$$

where the supremum is taken over the projections $Q$ of the form $Q_{1} \oplus Q_{2} \oplus Q_{3}$. This amounts to considering vectors $Z_{j}$ where only one of the three components is non-zero (and in fact of norm one in its space). Let then $m_{1}, m_{2}, m_{3}$, with $m_{1}+m_{2}+m_{3}=m$, be the numbers of vectors $Z_{j}$ of the form $(\Theta, 0,0),(0, X, 0)$, and $(0,0, Y)$, respectively. Applying Lemma 8.5, we get

$$
\operatorname{Tr}_{m}(M) \leq-m_{1}\left(\delta_{0} \ell m_{1}^{2 / 3}-c\right)-m_{2}\left(\delta_{0} \ell m_{2}^{2 / 3}-c\right)-\delta_{0} m_{3}
$$

which gives at once

$$
q_{m} \leq-m_{1}\left(\delta_{0} \ell m_{1}^{2 / 3}-c\right)-m_{2}\left(\delta_{0} \ell m_{2}^{2 / 3}-c\right)-\delta_{0} m_{3}
$$

Since as $m$ goes to infinity at least one of the $m_{i}$ 's goes to infinity, it is clear that there exists $m^{*}$ big enough such that $q_{m^{*}}<0$. Thus the desired conclusion follows from Theorem 8.3 and Proposition 8.4. 
Remark 8.7. With minor modifications, it is possible to extend the above results to a particular non-autonomous case (see, e.g., [GMP2]). Indeed, the uniform attractor $\mathcal{A}$ of the family $\left\{U_{f}(t, \tau), f \in \mathrm{H}(g)\right\}$ has finite fractal dimension, when the time-dependent function $g$ is quasiperiodic, namely, a function $g: \Omega \times \mathbb{R} \rightarrow \mathbb{R}$ of the form (see, e.g., $[\mathrm{AP}]$ )

$$
g(x, t)=G(x, \kappa t)=G\left(x, \kappa_{1} t, \ldots, \kappa_{m} t\right)
$$

where $G(\cdot, \varpi) \in C^{1}\left(\mathbf{T}^{m}, H\right)$ is a $2 \pi$-periodic function of $\varpi$ on the $m$-dimensional torus $\mathbf{T}^{m}$ and $\kappa=\left(\kappa_{1}, \ldots, \kappa_{m}\right)$ are rationally independent numbers. It is immediate to check that $g$ is translation compact in $L_{\mathrm{loc}}^{1}(\mathbb{R}, H)$, and $f \in \mathrm{H}(g)$ if and only if

$$
f(x, t)=G\left(x, \kappa t+\varpi_{0}\right) \quad \varpi_{0} \in \mathbf{T}^{m} .
$$

Therefore $\mathrm{H}(g)$ might be identified with $\mathbf{T}^{m}$, and the translation semigroup acting on $\mathrm{H}(g)$ is equivalent to the translation (modulus $2 \pi$ ) semigroup $R(t)$ on $\mathbf{T}^{m}$, defined by

$$
R(t) \varpi_{0}=\left[\kappa t+\varpi_{0}\right]=\kappa t+\varpi_{0} \quad \bmod 2 \pi .
$$

\section{REFERENCES}

[AB] S. Aizicovici $\mathcal{G}$ V. BARBu, Existence and asymptotic results for a system of integro-partial differential equations, NoDEA Nonlinear Differential Equations Appl. 3 (1996), 1-18.

[AP] L. Amerio $\mathcal{G}$ G. Prouse, Abstract Almost Periodic Functions and Functional Equations, Van Nostrand, New York, 1971.

[BV] A. V. Babin $\mathcal{E}$ M. I. VishiK, Attractors of Evolution Equations, North-Holland, Amsterdam, 1992.

[BL1] G. Bonfanti 63 F. Luterotti, Global solution to a phase-field model with memory and quadratic nonlinearity, Adv. Math. Sci. Appl. 9 (1999), 523-538.

[BL2] _ Regularity and convergence results for a phase-field model with memory, Math. Meth. Appl. Sci. 21 (1998), 1085-1105.

[Bre] H. BrÉzis, Opérateurs Maximaux Monotones et Semi-groupes de Contractions dans les Espaces de Hilbert, North-Holland Math. Stud., vol. 5, NorthHolland, Amsterdam, 1973.

[BS] M. Brokate 83 J. Sprekels, Hysteresis and Phase Transitions, Coll. Appl. Math. Sci., vol. 121, Springer, New York, 1996.

[Cag] G. Caginalp, An analysis of a phase field model of a free boundary, Arch. Rational Mech. Anal. 96 (1985), 205-245.

[CV1] V. V. Chepyzhov \& M. I. Vishik, Nonautonomous evolution equations and their attractors, Russian J. Math. Phys. 1 (1993), 165-190.

[CV2] - Attractors of non-autonomous dynamical systems and their dimension, J. Math. Pures Appl. 73 (1994), 279-333. 
[CV3] - Non-autonomous evolutionary equations with translation compact symbols and their attractor, C. R. Acad. Sci. Paris Sér. I Math. 321 (1995), 153-158.

[CG] B. D. Coleman ES M. E. Gurtin, Equipresence and constitutive equations for rigid heat conductors, Z. Angew. Math. Phys. 18 (1967), 199-208.

[CL1] P. Colli \& PH. LAurençOt, Existence and stabilization of solutions to the phase- field model with memory, J. Integral Equations Appl. 10 (1998), 168-194.

[CL2] Uniqueness of weak solutions to the phase-field model with memory, J. Math. Sci. Univ. Tokyo 5 (1998), 459-476.

[CGG1] P. Colli, G. Gilardi $\&$ M. Grasselli, Global smooth solution to the standard phase-field model with memory, Adv. Differential Equations 3 (1997), 453-486.

[CGG2] Well-posedness of the weak formulation for the phase-field model with memory, Adv. Differential Equations 3 (1997), 487-508.

[CGG3] Convergence of phase field to phase relaxation models with memory, Ann. Univ. Ferrara Ser. VII (N.S.), Suppl. 41 (1996), 1-14.

[CGG4] Asymptotic justification of the phase-field model with memory, Comm. Appl. Nonlinear Anal. 6 (1999), 1-27.

[CFT] P. Constantine, C. Foias 6 R. Temam, Attractors representing turbulent flows, 314, Mem. Amer. Math. Soc. 53 (1993).

[CH] R. Courant $\mathscr{G}$ D. Hilbert, Methods of Mathematical Physics, Intersciences, New York, 1953.

[Daf] C. M. Dafermos, Asymptotic stability in viscoelasticity, Arch. Rational Mech. Anal. 37 (1970), 297-308.

[FG] E. Fried \& M. E. GuRtin, Continuum theory of thermally induced phase transition based on an order parameter, Physica D 68 (1993), 326-343.

[GGP] C. Giorgi, M. Grasselli \& V. PATA, Well-posedness and longtime behavior of the phase-field model with memory in a history space setting, submitted.

[GMP1] C. Giongi, A. MARzocchi $\&$ V. PATA, Asymptotic behavior of a semilinear problem in heat conduction with memory, NoDEA Nonlinear Differential Equations Appl. 5 (1998), 333-354.

[GMP2] Uniform attractors for a non-autonomous semilinear heat equation with memory, to appear, Quart. Appl. Math.

[GP] M. Grasselli \& V. PATA, Longtime behavior of a homogenized model in viscoelastodynamics, Discrete Contin. Dynam. Systems 4 (1998), 339-358.

[GuP] M. E. Gurtin E A. C. Pipkin, A general theory of heat conduction with finite wave speeds, Arch. Rational Mech. Anal. 31 (1968), 113-126.

[HHM] B. I. Halperin, P. C. Hohenberg E S. K. MA, Renormalization-group methods for critical dynamics: I. Recursion relations and effects of energy conservation, Phys. Rev. B 10 (1974), 139-153.

[Har] A. Haraux, Systèmes Dynamiques Dissipatifs et Applications (Coll. RMA, ed.), vol. 17, Masson, Paris, 1990.

[Ken1] N. Kenмосні, Attractors of semigroups associated with nonlinear systems for diffusive phase separation, Abstr. Appl. Anal. 1 (1996), 169-192. 
[Ken2] G Global attractor of the semigroup associated to a phase-field model with constraint, Free Boundary Problems, Theory and Applications (Zakopane, 1995), vol. 363, Pitman Res. Notes Math. Ser., Longman, Harlow, 1996, pp. 111-125.

[KNZ] N. Kenmochi, M. Niezgódka $\&$ S. M. Zheng, Global attractor of the semigroup of a non-isothermal model for phase separation, Curvature Flows and Related Topics (Levico, 1994), Gakuto Internat. Ser. Math. Sci. Appl. 5, Gakkotosho, Tokyo, 1995, pp. 129-143.

[Lau] P. Laurençot, Long-time behaviour for a model of phase-field type, Proc. Roy. Soc. Edinburgh Sect. A 126 (1996), 167-185.

[PPV] V. Pata, G. Prouse 6 M. I. Vishik, Traveling waves of dissipative non-autonomous hyperbolic equations in a strip, Adv. Differential Equations 3 (1998), 249-270.

[PZ] V. PAta \& A AuCChI, Attractors for a damped hyperbolic equations with linear memory, submitted.

[PF] O. Penrose E P. C. Fife, Thermodynamically consistent models of phase-field type for the kinetics of phase transitions, Physica D 43 (1990), 44-62.

[SZ] J. Sprekels $\&$ S. Zheng, Maximal attractor for the system of a LandauGinzburg theory for structural phase transitions in shape memory alloys, Physica D 121 (1998), 252-262.

[Tem] R. Temam, Infinite-dimensional Dynamical Systems in Mechanics and Physics, Springer, New York, 1988.

[Vis] M. I. Vishiк, Asymptotic Behaviour of Solutions of Evolutionary Equations, Cambridge University Press, Cambridge, 1992.

This work has been partially supported by the Italian MURST '98 Research Projects "Modelli Matematici per la Scienza dei Materiali", "Equazioni Differenziali: Metodi Analitici, Geometrici e Funzionali, e Applicazioni", and "Problemi e Metodi nella Teoria delle Equazioni Iperboliche".

\section{Giorgi}

Dipartimento di Matematica

Università di Brescia

25133 Brescia, ITALY

EMAIL: giorgi@bsing.ing.unibs.it

M. Grasselli

Dipartimento di Matematica "F. Brioschi"

Politecnico di Milano, 20133 Milano, Italy

EMAIL: maugra@mate.polimi.it

V. PATA

Dipartimento di Matematica

Università di Brescia, 25133 Brescia, Italy

EMAIL: pata@bsing.ing.unibs.it 
SubJect Classification: 35B40, 35G25, 45K05, 80A22.

KEYWORDS: Phase-field models, materials with memory, existence and uniqueness, continuous dependence, non-autonomous dynamical systems, uniform absorbing sets, uniform attractors, Hausdorff and fractal dimensions.

Submitted: April 23rd, 1999. 